\title{
Les performances des entreprises françaises implantées à l'étranger
}

\author{
Alexandre Gazaniol*, Frédéric Peltrault* et Jean-Marc Siroen*
}

\begin{abstract}
Cet article évalue les performances des firmes multinationales dans l'industrie manufacturière française, et les compare à celles des entreprises uniquement exportatrices et domestiques. Les primes à l'implantation s'avèrent très significatives et dépassent largement les primes à l'exportation, indépendamment de la taille, de la conjoncture et du secteur d'activité. Cette hiérarchie se vérifie en tous points de la distribution conditionnelle de chaque variable par une régression quantile. Par ailleurs, les primes à l'implantation s'accroissent avec le nombre de filiales à l'étranger et sont plus marquées pour les filiales de groupe (en particulier étranger). Les meilleures performances des entreprises implantées à l'étranger s'expliquent en partie par un effet de sélection : investir à l'étranger s'accompagne de coûts fixes importants et nécessite un niveau de productivité relativement élevé. Toutefois, les firmes qui s'implantent pour la première fois à l'étranger, les primo-investisseurs, connaissent également une croissance significative de leurs ventes, de leur valeur ajoutée, de leurs effectifs et de leurs exportations ex post. L'implantation à l'étranger récompense ainsi les meilleures entreprises, qui consolident leur avantage par rapport aux autres firmes. Ce constat semble particulièrement vrai pour les filiales de groupes, qui se caractérisent à la fois par un effet de sélection et des effets d'apprentissage accrus.
\end{abstract}

\footnotetext{
* Université Paris-Dauphine, LEDa, F-75016 Paris, France et IRD, UMR225-DIAL, F-75010, Paris, France Nous tenons tout d'abord à remercier Matthieu Crozet ainsi que les rapporteurs anonymes pour leurs remarques qui ont considérablement contribué à l'amélioration de ce travail. Nous remercions également le cabinet de conseil Pramex International (Groupe BPCE), qui a été à l'initiative de cette recherche et l'a soutenue financièrement. Alexandre Gazaniol est employé par Pramex International dans le cadre d'une convention CIFRE passée avec l'Université de Paris Dauphine. Nous remercions par ailleurs Lionel Fontagné et Farid Toubal ainsi que tous les participants du groupe de travail du Conseil d'Analyse Economique pour leurs commentaires lors d'une présentation de ce travail. Enfin, nous remercions Laurence Germond et tous les services statistiques qui nous ont procuré les données et les informations nécessaires avec beaucoup de réactivité.
} 
$\grave{\mathbf{A}}$ l'occasion du lancement des États Généraux de l'industrie le 15 octobre 2009, le ministre de l'Industrie évoquait l'instauration d'une prime à la relocalisation de la production financée par l'État « en fonction des montants d'investissement et du volume d'emplois recréés ». En pleine crise économique, cette proposition met en exergue les craintes récurrentes associées à l'implantation à l'étranger des entreprises françaises : les investissements directs étrangers (IDE) sortants auraient un impact négatif sur la production et l'emploi domestiques. Pourtant, l'implantation à l'étranger joue un rôle important dans la fragmentation des processus de production qui expliquerait en partie les bonnes performances à l'exportation des entreprises allemandes (Fontagné et Gaulier, 2008).

Dans ce contexte, ce travail compare les performances des entreprises françaises implantées à l'étranger à celles des entreprises simplement exportatrices ou exclusivement domestiques. Nous savons déjà que les entreprises exportatrices sont plus performantes que les firmes limitées au marché national (Wagner, 2007). Les premières sont d'ailleurs relativement peu nombreuses et une minorité d'entre elles, les plus grandes, concentrent la plus grande part des exportations alors même qu'elles n'exportent souvent qu'une portion relativement faible de leur production. Ce constat n'est pas nouveau et avait déjà été dressé dans les années 1970 et 1980 par des auteurs intéressés par les effets de l'internationalisation sur les performances : effets d'échelle statique et dynamique, accès à l'innovation, contraintes concurrentielles $(X$-efficiency), effets de segmentation des marchés (1).

De nombreuses études récentes vérifient, en effet, les meilleures performances des entreprises exportatrices. Wagner (2007) recense ainsi 45 études micro-économétriques publiées entre 1995 et 2006 et portant sur 33 pays. Elles confirment l'existence d'une « prime à l'exportation » (écart de performance entre les firmes exportatrices et les firmes non exportatrices) (2). Ce constat s'applique à la France. Certaines études recensent les entreprises de manière quasi-exhaustive à partir de données douanières (Mayer et Ottaviano, 2007 ; Crozet et al., 2008 ; Eaton et al., 2004). D'autres utilisent les enquêtes annuelles d'entreprises (EAE) qui ne retiennent que les entreprises de plus de 20 salariés (Bellone et al. 2006, 2008 ; Isgep, 2008). Pour Crozet, Méjean et Zignago (2008), les exportateurs ont, en moyenne, une productivité supérieure de $11 \%$ et cette prime se retrouve dans la différence de salaire moyen et de taux de marge. Pour Bellone et al. (2008), les firmes exportatrices produisent 2,5 fois plus que les firmes simplement domestiques ; elles sont deux fois plus grandes, plus intensives en capital de $24 \%$ et versent des salaires plus élevés de $10 \%$. La productivité du travail serait supérieure de $40 \%$ mais décroissante avec la taille de l'entreprise. En termes de Productivité Totale des Facteurs (PTF), la prime des exportateurs, qui se situe autour de $5 \%$, tend à augmenter avec la part de la production exportée.

Si la littérature récente confirme les meilleures performances des firmes exportatrices, elle insiste sur l' « auto-sélection » à l'exportation : les bonnes performances et, plus précisément, un niveau de productivité plus élevé, précéderaient les exportations. La causalité inverse d'une influence positive des exportations sur les performances, suite à des effets d'apprentissage (3) ou des transferts de connaissances, n'est pas démontrée. De bonnes performances, en termes de productivité (productivité du travail et/ ou PTF), seraient ainsi un préalable et non une conséquence d'une stratégie d'exportation, ce qui n'exclut pas, toutefois, l'hypothèse selon laquelle cette amélioration des performances constituerait la première étape d'une stratégie d'internationalisation (Bellone et al., 2008). D'ailleurs, des travaux récents aboutissent à des résultats plus optimistes sur la présence d'effets d'apprentissage liés à l'activité d'exportation, notamment dans les pays en développement (Van Biesebrock, 2005, 2008 ; Isgut et Fernandes, 2007 ; Lileeva et Trefler, 2007 ; De Loecker, 2007). Les études montrent fréquemment que les firmes qui s'engagent à l'exportation connaissent une croissance plus rapide de la production et de l'emploi (Bernard et al, 2007).

Les performances supérieures des entreprises exportatrices et le sens de la causalité mis en évidence par ces travaux empiriques ont été

\footnotetext{
1. Pour l'état des lieux sur cette question dans les années 197080, voir Siroen (1985).

2. Voir également le survey de Greeenaway et Kneller (2007). Parmi les études par pays, citons Bernard et Jensen (1995, 1999, 2004a, 2004b) Bernard et al. (2003) pour les firmes américaines Bernard et Wagner (2001) et Wagner (2002) pour l'Allemagne; Aw et al. (2003) pour Taiwan et la Corée ; Clerides et al. (1998) pour la Colombie, le Mexique et le Maroc ; Girma et al. (2003, 2004) pour le Royaume-Uni. Yasar et al. (2006) appliquent la méthode des quantiles à des données turques.

3. Pour Clerides et al. (1998), les effets d'apprentissage liés à l'activité d'exportation résultent de l'expertise technique que les acheteurs étrangers procurent aux exportateurs. II en découle une amélioration de la productivité.
} 
théorisés par plusieurs auteurs et tout particulièrement par Melitz (2003). Le modèle proposé se place dans un cadre de concurrence monopolistique avec une différenciation horizontale des produits (4). Les firmes qui désirent exporter doivent supporter un coût fixe irrécouvrable et ignorent le niveau relatif de leur productivité dans la branche. Compte tenu de cette incertitude, seules les firmes bénéficiant d'un niveau acquis de productivité minimum (« export productivity cutoff ») prendront la décision d'exporter. Le modèle de Melitz établit donc un mécanisme d'auto-sélection pour expliquer la relation productivité-exportations telle qu'elle a été constatée dans les études empiriques.

Toutefois, le modèle de Melitz (2003) ne considère qu'une forme d'internationalisation des entreprises, l'exportation, négligeant deux autres grands modes d'expansion : les Investissements Directs Etrangers (IDE) et les licences. En se limitant à la prise en compte des IDE, Helpman et al. (HMY, 2004), à la suite de Brainard (1997), élargissent le modèle initial en intégrant les éléments de l'arbitrage entre les exportations et les ventes par les filiales locales. Ces deux modes d'accès au marché imposent des coûts relatifs différents. Les coûts irrécouvrables d'exportation incluent les coûts fixes de recherche, de réseaux de distribution ou de publicité, auxquels s'ajoutent des coûts variables comme les coûts de transport. Si l'investissement direct évite les coûts variables de transport, il supporte des coûts fixes spécifiques, supposés irrécouvrables, qui sont liés à la duplication des coûts dans les différents pays d'implantation. L'exportation est alors supposée moins coûteuse en termes de coûts fixes irrécouvrables, mais davantage en termes de coûts variables. Dans la logique du modèle d'《 auto-sélection » de Melitz, on s'attend donc à ce que les firmes les plus productives choisissent de s'implanter à l'étranger. En deçà d'un certain seuil, les firmes se contentent d'exporter en supportant des coûts irrécouvrables plus faibles. Comme dans le modèle initial, les firmes les moins productives ne s'internationalisent pas.

Comme le relèvent Greenaway et Kneller (2007), s'il existe une littérature abondante qui compare la productivité des firmes multinationales avec celles qui ne le sont pas (5), seulement un petit nombre d'études compare les performances des firmes exportatrices et des firmes multinationales, notamment parce que les données sont moins disponibles. Les études empiriques recensées par Greenaway et Kneller (2007) confirment l'hypothèse de la hiérarchie suggérée par le modèle de HMY avec néanmoins l'exception de Head et Ries (2003) pour le Japon, mais qui peut être attribuée à un échantillon limité à 1070 grandes firmes. Les travaux se sont néanmoins multipliés récemment. Tomiura (2007) utilise un échantillon de 118300 firmes japonaises pour vérifier que les firmes réalisant des IDE sont plus productives que les exportatrices (et que les entreprises qui externalisent une partie de leur activité à l'étranger) qui sont ellesmêmes plus productives que les firmes domestiques. Castellani et Zanfei (2007) constatent la meilleure productivité des firmes italiennes multinationales (qui ne sont pas, pour autant, plus innovatrices). Pour les secteurs du textilehabillement et de l'automobile-pièces détachées en Turquie, Yasar et Paul (2007) utilisent des régressions quantiles et des méthodes semiparamétriques. Ils confirment que les IDE sont davantage associés à une plus grande productivité que les exportations. Mayer et Ottaviano (2007) calculent les primes à l'exportation et à l'IDE pour 4 pays (France, Belgique, Norvège, et l'Allemagne uniquement pour l'emploi). Les primes à l'IDE sont nettement supérieures pour l'emploi et la valeur ajoutée. Cette supériorité est moins importante pour les salaires et l'intensité capitalistique (inversion pour la Norvège).

Dans la logique du modèle de HMY, le bonus de performance des entreprises françaises implantées à l'étranger provient tout d'abord d'un effet d'auto-sélection des entreprises les plus productives. L'application de techniques d'appariement ou "matching » permet de contrôler le biais de sélection et d'isoler les effets ex post de l'implantation à l'étranger. Le bilan global des études qui utilisent cette technique est que l'implantation à l'étranger n'a pas d'impact négatif sur les performances de la maison-mère. Kleinert et Toubal (2007) étudient les performances des primo-investisseurs sur données allemandes et trouvent un impact positif sur l'emploi domestique. Barba Navaretti et al. (2010) distinguent l'IDE dans les pays riches et dans les pays en développement à partir de données italiennes et françaises. Ils concluent que l'établissement d'unités de production dans les pays dits « low cost " (à bas coûts de main-d'œuvre) n'a pas d'impact négatif sur les activités de la maisonmère. Hijzen et al. (2009) distinguent IDE verticaux et horizontaux sur données françaises en analysant séparément l'industrie puis les servi-

4. Voir Bernard et al. (2007) pour l'introduction de cette approche dans le modèle « intégré » de Helpman et Krugman (1985). 5. Voir par exemple Doms et Jensen (1998) pour les États-Unis, Girma et al. (2001) pour le Royaume-Uni, Globerman et al. (1994) pour le Canada. 
ces. L'impact s'avère encore globalement positif sur l'emploi. En distinguant les entreprises selon leur appartenance à un groupe, Gazaniol et Peltrault (2010) constatent une augmentation de la productivité uniquement pour les filiales de groupes français.

\section{Analyse descriptive des performances des entreprises implantées à l'étranger}

L 'échantillon est obtenu en croisant deux sources d'information sur la période 20012007 : les enquêtes annuelles d'entreprise (EAE) pour l'industrie manufacturière, et l'enquête Liaisons Financières (LiFi) (cf. encadré 1).

En 2006, l'échantillon compte 1046 entreprises implantées à l'étranger, détenant ensemble 4402 implantations à l'étranger. Quelle que soit leur taille, ces entreprises ont en commun d'opter largement pour des prises de participation majoritaires : elles préfèrent donc s'assurer un contrôle exclusif de leurs filiales à l'étranger. De manière attendue, la proportion d'entreprises effectuant des IDE augmente avec les effectifs (cf. tableau 1). Ainsi, les entreprises de plus de 250 salariés représentent seulement $9 \%$ des entreprises de l'échantillon mais $49 \%$ des entreprises implantées à l'étranger. De plus, les grandes entreprises disposent d'un réseau d'im-

Encadré 1

\section{LES DONNÉES}

L'échantillon est obtenu en croisant deux sources d'information sur la période 2001-2007 : les enquêtes annuelles d'entreprise (EAE) pour l'industrie manufacturière, et l'enquête Liaisons Financières ( $L i F i)$. Les $E A E$ fournissent un compte de résultat détaillé pour toutes les entreprises industrielles de plus de 20 salariés (environ 20000 entreprises par année en moyenne). Elles renseignent sur leur chiffre d'affaires, leurs effectifs, leurs immobilisations, leur valeur ajoutée, leurs investissements et leurs exportations. L'enquête LiFi permet quant à elle d'identifier les entreprises qui réalisent des Investissements Directs à l'Etranger (IDE), c'est-à-dire qui s'implantent à l'étranger sous la forme de filiales ou de prises de participation au capital de sociétés étrangères (conformément à la définition officielle des IDE, nous ne retenons que les prises de participation supérieures à $10 \%$ du capital). L'enquête LiFi permet également de connaître la structure de l'actionnariat des entreprises françaises, que nous pouvons alors séparer en quatre catégories : les entreprises têtes de groupe (une tête de groupe est une entreprise non contrôlée directement ou indirectement par une autre entreprise, et qui a au moins une filiale), les entreprises contrôlées par un groupe français, les entreprises contrôlées par un groupe étranger et les entreprises indépendantes.

Le croisement des enquêtes annuelles d'entreprise et de l'enquête LiFi nous permet d'identifier trois types d'entreprises, conformément à la littérature :

- les entreprises domestiques, qui n'ont aucune activité à l'international ;

- les entreprises exportatrices, sans implantation à l'étranger :

- les entreprises françaises implantées à l'étranger. Notons que $98 \%$ des entreprises implantées à l'étranger de notre échantillon sont également exportatrices.
Le seuil de 20 salariés des enquêtes annuelles d'entreprise conduit à surreprésenter les entreprises exportatrices et implantées à l'étranger. Les firmes exportatrices composent en effet $70 \%$ de l'échantillon, les entreprises implantées à l'étranger $6 \%$. À titre de comparaison, en utilisant la base des Bénéfices Réels Normaux, qui intègre les firmes quelle que soit leur taille, Crozet et al. (2008) évaluent la représentation des firmes exportatrices à $21 \%$.

La base de données est « nettoyée » en suivant quatre étapes, au cours desquelles sont retirées :

- les observations affiliées au secteur énergétique, afin de nous restreindre au champ de l'industrie manufacturière ;

- les entreprises qui changent de secteur d'activité (Nomenclature Économique de Synthèse (NES) à 36 niveaux) durant la période 2001-2006 ;

- les valeurs manquantes, nulles ou négatives pour le chiffre d'affaires, la valeur ajoutée, l'emploi, les salaires, les immobilisations, et l'investissement corporel, afin d'utiliser un seul et même échantillon dans tout notre travail. Les différences de résultats entre plusieurs modèles peuvent entièrement être attribuées à un changement de spécification du modèle ou d'une variable, et non à un changement du nombre d'observations utilisées.

- les valeurs extrêmes pour la productivité du travail et l'intensité capitalistique en suivant la méthodologie de Hall et Mairesse (1995). En effet, la présence de ces valeurs dans l'échantillon peut considérablement biaiser l'estimation de nos modèles économétriques. Les retirer réduit nettement le niveau des primes mais ne modifie pas les conclusions de nos travaux.

Ce « nettoyage » retire environ $17 \%$ des observations de l'échantillon initial. 
plantations à l'étranger beaucoup plus dense que les entreprises de moins de 250 salariés : les premières peuvent s'appuyer sur plus de six implantations en moyenne contre une à deux implantations pour les secondes.

\section{Une propension à s'implanter à l'étranger différente selon les secteurs}

La répartition entre entreprises domestiques, exportatrices et implantées à l'étranger s'avère très hétérogène selon les secteurs d'activités (cf. tableau 2). Le secteur le plus internationalisé de l'échantillon est « pharmacie, parfums et entretien » avec seulement $9 \%$ d'entreprises domestiques et plus de $17 \%$ de firmes implantées à l'étranger, chacune comptant en moyenne neuf implantations à l'étranger. Alors que ce secteur pèse à peine $3 \%$ de l'échantillon en 2006 , il représente $7 \%$ du nombre total de multinationales et $15 \%$ du nombre total d'implantations à l'étranger. Le cas de figure est exactement inverse pour le secteur de l'édition, imprimerie

Tableau 1

Nombre d'entreprises implantées à l'étranger et d'investissements directs étrangers (IDE) selon la classe d'effectifs en 2006

\begin{tabular}{|l|c|c|c|c|c|}
\hline \multicolumn{1}{|c|}{ Classe d'effectifs } & $\begin{array}{c}\text { Nombre } \\
\text { d'entreprises }\end{array}$ & $\begin{array}{c}\text { dont implantées } \\
\text { à l'étranger }\end{array}$ & $\begin{array}{c}\text { Nombre } \\
\text { d'implantations } \\
\text { à l'étranger }\end{array}$ & $\begin{array}{c}\text { dont prises } \\
\text { de participation } \\
\text { majoritaires }\end{array}$ & $\begin{array}{c}\text { dont prises } \\
\text { de participation } \\
\text { minoritaires }\end{array}$ \\
\hline Moins de 50 salariés & 8774 & 117 & 163 & 125 & 38 \\
50 - 249 salariés & 5972 & 420 & 943 & 768 & 643 \\
250 - 499 salariés & 814 & 204 & 2538 & 2175 & 363 \\
500 salariés et plus & 647 & 305 & 4402 & 3711 & 691 \\
\hline Toutes & 16207 & 1046 & 758 & \\
\hline
\end{tabular}

Lecture: dans l'échantillon, 117 entreprises de moins de 50 salariés sont implantées à l'étranger en 2006. L'ensemble des entreprises de moins de 50 salariés a 163 implantations à l'étranger.

Champ : industrie manufacturière, entreprises de plus de 20 salariés.

Sources : EAE, LiFi - Calcul des auteurs.

Tableau 2

Répartition des entreprises domestiques, exportatrices et implantées à l'étranger selon le secteur d'activité

\begin{tabular}{|c|c|c|c|c|c|c|c|}
\hline $\begin{array}{l}\text { Secteur d'activité } \\
\text { (NES à } 36 \text { niveaux) }\end{array}$ & $\begin{array}{c}\text { Total } \\
\text { d'entreprises }\end{array}$ & $\begin{array}{c}\text { Entreprises } \\
\text { domestiques }\end{array}$ & $\begin{array}{l}\text { Entreprises } \\
\text { exportatrices }\end{array}$ & $\begin{array}{l}\text { Entreprises } \\
\text { implantées } \\
\text { à l'étranger }\end{array}$ & $\begin{array}{c}\text { Total } \\
\text { d'implantations } \\
\text { à l'étranger }\end{array}$ & $\begin{array}{l}\text { Nombre } \\
\text { de PME }\end{array}$ & $\begin{array}{c}\text { PME } \\
\text { implantées } \\
\text { à l'étranger }\end{array}$ \\
\hline Habillement et Cuir & 632 & 117 & 448 & 67 & 253 & 602 & 45 \\
\hline $\begin{array}{l}\text { Édition, imprimerie et } \\
\text { reproduction }\end{array}$ & 1243 & 442 & 766 & 35 & 129 & 1168 & 16 \\
\hline $\begin{array}{l}\text { Pharmacie, Parfums et } \\
\text { Entretien }\end{array}$ & 431 & 38 & 319 & 74 & 646 & 315 & 22 \\
\hline Équipements du Foyer & 979 & 179 & 726 & 74 & 357 & 883 & 44 \\
\hline Industrie Automobile & 427 & 53 & 349 & 25 & 93 & 324 & 3 \\
\hline $\begin{array}{l}\text { Construction Navale, } \\
\text { Aéronautique et Ferroviaire }\end{array}$ & 235 & 43 & 169 & 23 & 93 & 191 & 7 \\
\hline Équipements Mécaniques & 3016 & 892 & 1940 & 184 & 624 & 2808 & 107 \\
\hline $\begin{array}{l}\text { Équipements Électriques/ } \\
\text { Électroniques }\end{array}$ & 854 & 200 & 563 & 91 & 336 & 779 & 57 \\
\hline Produits Minéraux & 1103 & 553 & 500 & 50 & 195 & 1010 & 21 \\
\hline Textile & 680 & 91 & 555 & 34 & 88 & 642 & 22 \\
\hline Bois et Papier & 1046 & 364 & 644 & 38 & 109 & 958 & 11 \\
\hline $\begin{array}{l}\text { Chimie, Caoutchouc et } \\
\text { Plastiques }\end{array}$ & 1816 & 283 & 1362 & 171 & 674 & 1611 & 101 \\
\hline Métallurgie & 3082 & 709 & 2266 & 107 & 335 & 2896 & 53 \\
\hline $\begin{array}{l}\text { Composants Électriques/ } \\
\text { Électroniques }\end{array}$ & 663 & 106 & 484 & 73 & 470 & 559 & 28 \\
\hline Tous secteurs & 16207 & 4070 & 11091 & 1046 & 4402 & 14746 & 537 \\
\hline
\end{tabular}

Lecture : en 2006, la métallurgie compte 107 entreprises implantées à l'étranger (avec une moyenne de 3 implantations par entreprise), dont 53 sont des $P M E$.

Champ : industrie manufacturière, entreprises de plus de 20 salariés.

Sources : EAE, LiFi - Calcul des auteurs. 
et reproduction, qui compte $36 \%$ d'entreprises domestiques et seulement $3 \%$ d'entreprises implantées à l'étranger. Malgré un poids de $8 \%$ dans l'échantillon, ce secteur abrite seulement $3 \%$ du nombre total de multinationales.

Plusieurs arguments théoriques peuvent expliquer ces différences entre secteurs : le degré d'hétérogénéité des firmes, le degré de différenciation des produits, l'importance des coûts de transport ou encore la complexité et la « séparabilité » des chaînes de production. Les industries les plus pourvoyeuses d'IDE sont en effet celles où la différenciation des produits est fine (chimie et pharmacie par exemple), ce qui incite les entreprises à rapprocher leur production et leurs activités de R\&D des consommateurs, et celles dont la chaîne de production peut être éclatée géographiquement afin de chercher des avantages de coûts. Ce deuxième cas de figure concerne notamment les produits à faible contenu technologique (habillement et cuir, textile, automobile pour les modèles de bas/moyenne gamme (6), moins liés à des contraintes de qualification du personnel ou de contrôle de la qualité. Toutefois, la plupart des secteurs s'inscrivent à la fois dans une logique de conquête de marchés et de réduction des coûts (7) : c'est notamment le cas de l'industrie automobile, qui s'est en partie relocalisée dans les Pays d'Europe Centrale et Orientale (PECO) afin non seulement de béné- ficier de coûts de production plus faibles, mais aussi d'être présente sur des marchés à forte croissance. L'internationalisation de certaines industries est par ailleurs liée à leurs donneurs d'ordres : ces derniers qui souhaitent fonctionner en flux tendus incitent leurs fournisseurs à les suivre à l'étranger (voir les équipementiers automobiles et l'industrie mécanique). Enfin, la motivation de l'implantation à l'étranger peut être influencée par les caractéristiques des produits : dans le secteur de la chimie de base, la dangerosité du transport et du stockage de certaines matières peut contraindre les producteurs à s'implanter à l'étranger.

\section{Les entreprises implantées à l'étranger sont souvent elles-mêmes des filiales de groupe}

En 2006, $80 \%$ des entreprises implantées à l'étranger sont contrôlées par un groupe, contre $59 \%$ des firmes exportatrices et $48 \%$ des firmes domestiques. Par ailleurs, la proportion d'entreprises implantées à l'étranger qui sont têtes de groupe diminue sensiblement avec l'effectif (cf. graphique I). En effet, $50 \%$ des entreprises implantées à l'étranger de moins de 50 salariés sont des têtes

6. Voir Bouabdallah et al. (2008).

7. Voir Pliquet et Riedinger (2008).

\section{Graphique I}

\section{Appartenance à un groupe des entreprises implantées à l'étranger selon leur taille en 2006}

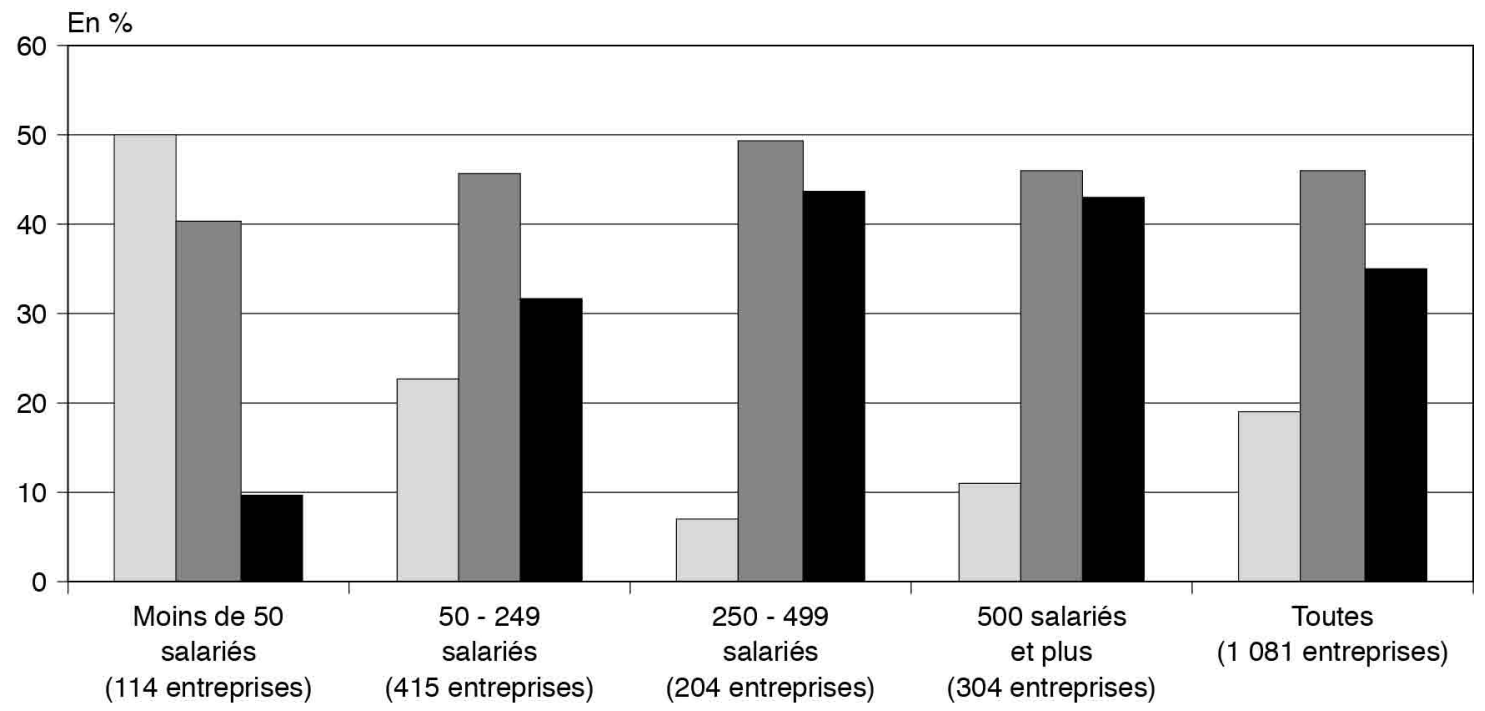

$\square$ Têtes de groupe

$\square$ Contrôlées par un groupe français

Contrôlées par un groupe étranger

Lecture : en 2006, 43 \% des entreprises implantées à l'étranger de plus de 500 salariés sont contrôlées par un groupe étranger. Par souci de simplification, les entreprises communes et les entreprises dans la mouvance ou le contour élargi de groupes ont été retirées de l'échantillon (9 entreprises retirées au total).

Champ : industrie manufacturière, entreprises de plus de 20 salariés.

Sources : EAE, LiFi - Calcul des auteurs. 
de groupe, contre seulement $10 \%$ des entreprises implantées à l'étranger de plus de 250 salariés.

Ce constat tient au fait qu'il existe une corrélation négative entre la taille et le degré d'indépendance. En effet, les grandes sociétés ont souvent besoin d'entrer dans un groupe pour poursuivre leur développement. De plus, les groupes préfèrent absorber des sociétés déjà opérationnelles, très productives et tournées vers les marchés extérieurs, afin d'économiser des coûts fixes (R\&D, acheminement des produits, distribution, infrastructures, etc.) (8).

Toutefois, si grandir accroît la probabilité d'être absorbé par un groupe, le sens de la causalité peut également être inversé. En effet, l'appartenance à un groupe peut impliquer des ressources humaines et financières accrues ainsi que l'accès à de nouveaux marchés. Pour les entreprises implantées à l'étranger, le soutien d'un groupe peut également procurer une connaissance accrue des marchés extérieurs et de la culture locale, une visibilité internationale renforcée, de bonnes pratiques en termes d'implantation, ou encore révéler des synergies avec d'autres filiales basées à l'étranger. Il peut donc être intéressant de distinguer les performances des entreprises implantées à l'étranger selon leur appartenance à un groupe.

\section{Les entreprises implantées à l'étranger contribuent beaucoup plus que les autres à l'activité économique}

La littérature économique établit une hiérarchie entre les firmes selon leur mode d'entrée sur les marchés extérieurs (cf. supra) : IDE, exportations, marché domestique. Cette hiérarchie se vérifie sur des données françaises, en considérant plusieurs indicateurs de performances (la productivité, le chiffre d'affaires, la valeur ajoutée, l'intensité d'exportation c'est-à-dire la part des exportations dans le chiffre d'affaires, l'emploi, le salaire moyen) (9). Nous ne disposons d'informations comptables que sur la partie française des groupes : seules les performances des entreprises (unités légales) localisées en France sont donc étudiées ici, et non celles des groupes dans leur ensemble.

Il apparaît tout d'abord que les entreprises implantées à l'étranger contribuent de manière disproportionnée à la production, à la valeur ajoutée, à l'emploi et aux exportations (cf. graphique II). Alors qu'elles ne représentent ensem-

8. À ce propos, voir Nefussi (2007).

9. Toutes les variables d'intérêt sont déflatées en utilisant des séries fournies par l'Insee (année de base 2001).

\section{Graphique II \\ Partage de l'activité économique selon le statut à l'international et l'appartenance à un groupe en 2006}

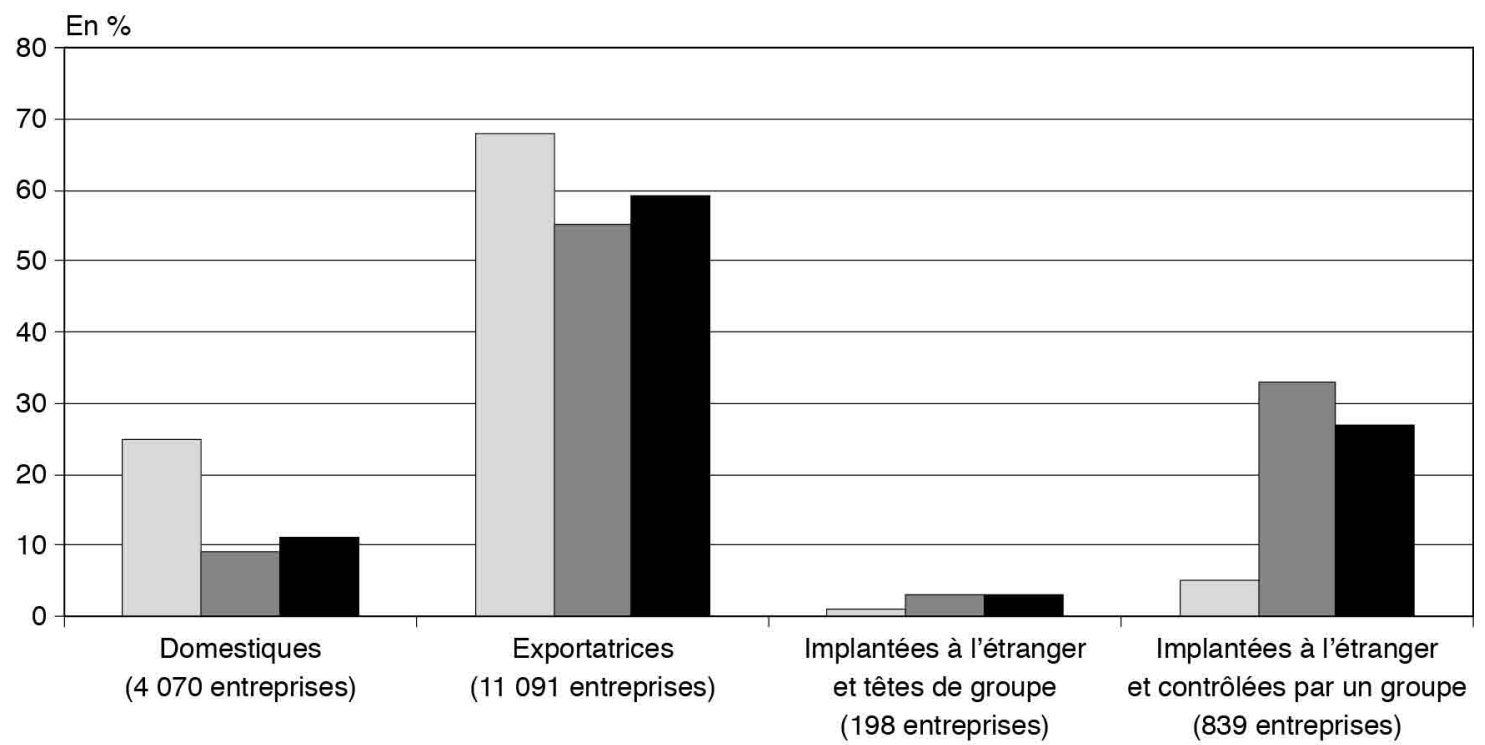


ble que $6 \%$ des entreprises industrielles, elles réalisent $38 \%$ du chiffre d'affaires, $36 \%$ de la valeur ajoutée, $51 \%$ des exportations et regroupent $30 \%$ de l'emploi. En moyenne, chaque entreprise implantée à l'étranger exporte ainsi 60 millions d'euros et compte 582 employés.

Toutefois, cette disproportion entre poids dans l'échantillon et poids dans l'activité concerne surtout les entreprises implantées à l'étranger qui sont filiales d'un groupe. En effet, elles ne représentent que $5 \%$ de l'échantillon, mais près d'un tiers du chiffre d'affaires et de la valeur ajoutée, plus du quart de l'emploi et presque la moitié des exportations. En revanche, les têtes de groupe implantées à l'étranger ont une contribution à l'activité plus conforme à leur poids dans l'échantillon.

\section{Une hiérarchie vérifiée au sein de chaque classe d'effectif et chaque secteur}

Le poids des entreprises implantées à l'étranger dans l'économie n'est pas uniquement le fait de quelques grandes multinationales. Les PME qui disposent de filiales à l'étranger jouissent également de performances très supérieures aux autres PME (cf. tableau 3). De manière plus surprenante, les performances moyennes des entreprises implantées à l'étranger sont relativement comparables d'une classe d'effectif à une autre en termes de productivité du travail, de salaires versés ou encore d'intensité d'exportation. Par exemple, les multinationales de moins de 50 salariés ont le même niveau moyen de productivité que les multinationales de plus de 250 salariés. Au sein de ces entreprises, certains indicateurs de performances semblent donc peu sensibles au facteur « taille ».

Cette hiérarchie au sein de chaque classe d'effectif se retrouve également au sein de chaque secteur d'activité (cf. tableau A en annexe 1). Cette fois encore, les résultats sont hétérogènes selon les secteurs, notamment en matière de productivité, de salaires versés ou encore d'intensité d'exportation. Par exemple, une multinationale du secteur de l'édition, imprimerie et reproduction exporte $17 \%$ de son chiffre d'affaires, contre $44 \%$ pour une entreprise du secteur de la pharmacie, parfumerie, entretien.

L'implantation à l'étranger semble donc favoriser les exportations, au sein de chaque secteur par classe d'effectif. Cette complémentarité entre IDE et exportations se manifeste tout à la fois par un chiffre d'affaires à l'export et une intensité d'exportation plus élevés pour les entreprises implantées à l'étranger que pour les entreprises exportatrices.

\section{Primes à l'exportation et à l'implantation}

près une analyse descriptive des performances relatives des entreprises implantées à l'étranger, nous estimons maintenant

Tableau 3

Performances moyennes des entreprises industrielles selon leur statut à l'international et leur classe d'effectif en 2006

\begin{tabular}{|c|c|c|c|c|c|c|c|c|}
\hline $\begin{array}{l}\text { Classe } \\
\text { d'effectif }\end{array}$ & $\begin{array}{c}\text { Statut à } \\
\text { l'international }\end{array}$ & $\begin{array}{c}\text { Nombre } \\
\text { d'entreprises }\end{array}$ & $\begin{array}{c}\text { Chiffre } \\
\text { d'affaires } \\
\text { en milliers } \\
\text { d'euros }\end{array}$ & $\begin{array}{l}\text { Valeur } \\
\text { ajoutée } \\
\text { en milliers } \\
\text { d'euros }\end{array}$ & Effectifs & $\begin{array}{l}\text { Ratio } \\
\text { valeur } \\
\text { ajoutée/ } \\
\text { effectif }\end{array}$ & $\begin{array}{c}\text { Salaire } \\
\text { mensuel brut } \\
\text { en milliers } \\
\text { d'euros }\end{array}$ & $\begin{array}{c}\text { Intensité } \\
\text { d'exportation } \\
\text { (en \%) }\end{array}$ \\
\hline \multirow{3}{*}{$\begin{array}{l}\text { Moins de } \\
50 \text { salariés }\end{array}$} & Domestique & 2944 & 3768 & 1630 & 32 & 52 & 1,97 & 0 \\
\hline & Exportatrice & 5713 & 4873 & 1890 & 33 & 57 & 2,13 & 18 \\
\hline & Implantée à l'étranger & 117 & 8652 & 2735 & 36 & 79 & 2,66 & 38 \\
\hline \multirow{3}{*}{$\begin{array}{l}50 \text { - } 249 \\
\text { salariés }\end{array}$} & Domestique & 1048 & 12102 & 4637 & 91 & 51 & 1,85 & 0 \\
\hline & Exportatrice & 4504 & 15798 & 5903 & 105 & 56 & 2,02 & 23 \\
\hline & Implantée à l'étranger & 420 & 29120 & 10069 & 137 & 74 & 2,47 & 43 \\
\hline \multirow{3}{*}{$\begin{array}{l}250-499 \\
\text { salariés }\end{array}$} & Domestique & 55 & 53665 & 18603 & 338 & 57 & 2,11 & 0 \\
\hline & Exportatrice & 555 & 60615 & 20477 & 339 & 60 & 2,08 & 31 \\
\hline & Implantée à l'étranger & 204 & 75768 & 26605 & 349 & 77 & 2,55 & 41 \\
\hline \multirow{3}{*}{$\begin{array}{c}\text { Plus de } \\
500 \text { salariés }\end{array}$} & Domestique & 23 & 120375 & 47617 & 760 & 64 & 2,33 & 0 \\
\hline & Exportatrice & 319 & 240740 & 80778 & 1066 & 72 & 2,41 & 35 \\
\hline & Implantée à l'étranger & 305 & 397364 & 130473 & 1563 & 78 & 2,58 & 42 \\
\hline
\end{tabular}

Lecture : en 2006, les entreprises implantées à l'étranger de moins de 50 salariés ont un chiffre d'affaires moyen de 8,652 millions d'euros et une intensité d'exportation moyenne de $38 \%$.

Champ : industrie manufacturière, entreprises de plus de 20 salariés.

Sources : EAE, LiFi - Calcul des auteurs. 
les primes à l'exportation et à l'implantation à l'aide d'un modèle économétrique. La prime à l'exportation est définie comme le bonus de performances qu'affichent les exportateurs par rapport aux firmes domestiques. De la même façon, la prime à l'implantation est définie comme le bonus de performances qu'affichent les entreprises implantées à l'étranger par rapport aux firmes domestiques (cf. le modèle économétrique utilisé pour calculer ces primes dans l'encadré 2).

\section{Les primes à l'implantation dépassent largement les primes à l'exportation, en tous points de la distribution.}

Il apparaît que toutes les primes sont positives et significatives, et que les primes à l'implan- tation sont supérieures aux primes à l'exportation (cf. tableau 4). Ce résultat reste vérifié en contrôlant l'hétérogénéité non observée par des effets fixes, même si le niveau des primes s'en trouve considérablement réduit (cf. tableau B en annexe 1). C'est sur les effectifs que la prime à l'implantation est la plus élevée. La prime de chiffre d'affaires s'accompagne d'une prime de valeur ajoutée : les entreprises implantées à l'étranger ne se contentent donc pas de revendre une production réalisée à l'étranger, mais se caractérisent bien par une contribution au PIB plus importante. Elles rémunèrent mieux leur personnel, ce qui reflète probablement un niveau de qualification plus élevé. Enfin, la prime de productivité pour les entreprises implantées à l'étranger représente le triple de celle calculée pour les firmes exportatrices.

Encadré 2

\section{L'ESTIMATION DES PRIMES À L'EXPORTATION ET À L'IMPLANTATION}

Les primes à l'exportation et à l'implantation sont d'abord estimées par la méthode des Moindres Carrés Ordinaires (MCO), avec l'équation de régression suivante :

$$
\begin{aligned}
& \ln X_{i t}=\alpha+\beta_{1} \times \text { Export }+\beta_{2} \times \text { Ide }+\lambda \text { InEff }_{i t} \\
& +\sum_{j} \delta_{1 j} \times S_{j}+\sum_{t} \delta_{2 t} \times A_{t}+\varepsilon_{i t} \text { (1) }
\end{aligned}
$$

$X$ désigne la caractéristique étudiée de la firme : successivement la productivité totale des facteurs (cf. annexe 2), la productivité du travail, l'emploi, le chiffre d'affaires, la valeur ajoutée, et les salaires. La variable Export prend la valeur 1 si l'entreprise est exportatrice (sans implantation à l'étranger), 0 sinon. La variable Ide prend la valeur 1 si l'entreprise est implantée à l'étranger, 0 sinon. Les primes à l'exportation et à l'implantation sont donc respectivement mesurées par les paramètres $\beta_{1}$ et $\beta_{2}$. La variable Eff représente l'effectif employé ; elle vise à prendre en compte la taille de l'entreprise, qui elle-même, détermine en partie la taille et le montant des opérations engagées à l'international. Cette variable est retirée du modèle lorsque l'emploi ou la productivité du travail deviennent la variable expliquée. Les variables $S$ et $A$ contrôlent respectivement le secteur d'activité $S_{i}, j=\{1, \ldots, 14\}$, et l'année $A_{t}, t=\{2001, \ldots, 2006\}$. La dimension dynamique de notre panel permet par ailleurs d'introduire des effets fixes au niveau des firmes, pour prendre en compte des caractéristiques inobservables mais supposées invariantes dans le temps (comme l'organisation, le savoir-faire etc.). Enfin, pour vérifier si les différences entre coefficients d'un même modèle sont significatives, nous réalisons plusieurs tests d'égalité des coefficients, en estimant l'écart-type avec des techniques de bootstrapping (50 réplications).
En présence d'une forte hétérogénéité entre les entreprises, il est possible que l'estimation par les MCO, centrée sur la firme moyenne, donne une image très imparfaite des primes. D'une part, celles-ci peuvent varier d'un bout à l'autre de la distribution de la variable dépendante. D'autre part, la moyenne est très influencée par les valeurs extrêmes de l'échantillon. C'est pourquoi nous estimons également le modèle ci-dessus avec la régression quantile, introduite par Koenker et Basset $(1978,2001)$, qui prend en compte l'hétérogénéité de la variable dépendante. Cette méthode permet d'exprimer les quantiles de la distribution conditionnelle en fonction des variables explicatives. Soit $Q_{\theta}\left(x_{i t} / z_{i t}\right)$ le quantile d'ordre $\theta$ relatif aux entreprises ayant les caractéristiques $z$ et $\beta_{\theta}$ le vecteur des coefficients tel que :

$\mathrm{Q}_{\theta}\left(\mathrm{x}_{\mathrm{it}} / \mathrm{z}_{\mathrm{it}}\right)=\mathrm{z}_{\mathrm{it}}{ }^{\mathrm{t}} \beta_{\theta}$

Les coefficients de la régression quantile sont alors estimés par le programme d'optimisation suivant :

$\operatorname{Min}_{\beta}\left\{\sum_{i, t: x_{i t} \geq z_{i t}{ }^{\prime} \beta} \theta\left|x_{i t}-z_{i t}{ }^{\prime} \beta\right|+\sum_{i, t: x_{i t}<z_{i t}{ }^{\prime} \beta}(1-\theta)\left|x_{i t}-z_{i t}{ }^{\prime} \beta\right|\right\}$

La méthode de la régression quantile s'appuie ainsi sur l'ensemble de l'échantillon, en pondérant davantage certaines observations que d'autres en fonction de leur position sur la distribution conditionnelle de la variable dépendante (en ce sens, la régression quantile est différente d'une régression linéaire effectuée uniquement sur les points du quantile d'ordre $\theta$ qui rencontrerait un biais de sélection). Les coefficients $\beta_{\theta}$ estimés sont alors différents pour chaque quantile. Ils peuvent s'interpréter comme l'effet de la variation marginale d'une variable explicative $z$ sur la variable dépendante $x$ au quantile. 
La hiérarchie entre entreprises domestiques, exportatrices et implantées à l'étranger est vérifiée en tous points de la distribution, comme le montrent les résultats de la régression quantile (cf. tableau $C$ en annexe 1) (10). Les primes ont tendance à s'élever dans les derniers quantiles de la distribution : autrement dit, pour chaque variable, l'écart entre les exportateurs les plus performants et les multinationales les plus performantes est supérieur à l'écart entre les exportateurs les moins performants et les multinationales les moins performantes. Seule exception, la prime de salaires reste relativement stable : quel que soit le quantile considéré, les entreprises exportatrices et implantées à l'étranger rémunèrent respectivement $8 \%$ et $24 \%$ mieux qu'une entreprise domestique.

\section{Des primes croissantes avec le nombre de filiales à l'étranger}

Pour distinguer les primes à l'implantation selon la dimension du réseau à l'international, nous scindons la variable Ide du premier modèle en quatre variables IdeI, Ide2, Ide 3 et Ide5, qui prennent la valeur 1 si l'entreprise a respectivement une, deux, entre trois et cinq, et plus de cinq implantations à l'étranger (11).

Les primes à l'implantation s'accroissent nettement avec la taille de leur réseau à l'international (cf. tableau 5). En effet, multiplier les implantations à l'étranger engendre des coûts supplémentaires, qui demandent à chaque fois un niveau de productivité plus élevé. Toutefois, il est intéressant de noter que les primes des entreprises n'ayant qu'une seule filiale à l'étranger sont déjà élevées, même très proches des primes à l'implantation moyennes. La première implantation à l'étranger semble donc déjà constituer un cap difficile, seulement accessible à un club d'entreprises très restreint.

\section{Effet de sélection et amélioration des performances ex post}

Les primes à l'implantation s'expliquent en partie par un effet de sélection. De nombreux travaux empiriques mettent ainsi en évidence qu'un haut niveau de productivité accroît sensiblement la probabilité de s'implanter à l'étranger (Barba Navaretti et al., 2010 ; Kleinert et Toubal, 2007). Sur les mêmes données et une période très proche (1999-2007), Gazaniol et Peltrault (2010) montrent que cette probabilité est fonction croissante du nombre d'établissements, du chiffre d'affaires, de la productivité et de l'intensité d'exportation (cf. tableau 6).

Les mêmes auteurs démontrent également une amélioration des performances ex post, en s'appuyant sur des méthodes d'appariement (ou « matching »). Ces méthodes consistent à comparer les firmes qui s'implantent à l'étranger pour la première fois, les primo-investisseurs, à des firmes domestiques présentant les mêmes caractéristiques (taille, productivité, secteur, cohorte, degré d'indépendance). L'impact des IDE est mesuré par l'estimateur " double différence », qui mesure l'évolution de l'écart de performances entre firmes domestiques et primo-investisseurs, entre la période ex ante et la période ex post. Il permet ainsi de contrôler l'influence de caractéristiques non observables des firmes, mais jugées invariantes dans le temps, comme le savoir-faire

10. En raison de la lourdeur du calcul, la régression quantile n'a pas pu être estimée en intégrant des effets fixes firmes.

11. Ces seuils de une, deux, trois puis cinq implantations sont notamment choisis afin d'obtenir quatre classes d'entreprises de taille équivalente.

Tableau 4

Primes à l'exportation et à l'implantation dans l'industrie manufacturière (période 2001-2006)

\begin{tabular}{|c|c|c|c|c|c|c|}
\hline Statut à l'international & $\begin{array}{c}\text { Productivité } \\
\text { Totale } \\
\text { des Facteurs }\end{array}$ & $\begin{array}{c}\text { Productivité } \\
\text { du travail }\end{array}$ & $\begin{array}{c}\text { Chiffre } \\
\text { d'affaires }\end{array}$ & $\begin{array}{l}\text { Valeur } \\
\text { ajoutée }\end{array}$ & Effectifs & $\begin{array}{l}\text { Salaire } \\
\text { moyen }\end{array}$ \\
\hline Prime à l'exportation (1) & $\begin{array}{c}0,0621^{\text {**}} \\
(0,00456)\end{array}$ & $\begin{array}{c}0,0927^{\text {**}} \\
(0,00474)\end{array}$ & $\begin{array}{c}0,231^{\star \star *} \\
(0,00712)\end{array}$ & $\begin{array}{c}0,0937^{\text {**}} \\
(0,00485)\end{array}$ & $\begin{array}{c}0,371^{\star \star \star} \\
(0,00988)\end{array}$ & $\begin{array}{c}0,0808^{\star \star *} \\
(0,00324)\end{array}$ \\
\hline Prime à l'implantation (2) & $\begin{array}{l}0,218^{\star \star \star} \\
(0,0121)\end{array}$ & $\begin{array}{c}0,275^{\star \star \star} \\
(0,0107)\end{array}$ & $\begin{array}{l}0,519^{\star \star \star} \\
(0,0165)\end{array}$ & $\begin{array}{l}0,280^{\star \star \star} \\
(0,0119)\end{array}$ & $\begin{array}{l}1,809^{\star \star \star} \\
(0,0337)\end{array}$ & $\begin{array}{c}0,237^{\star \star \star} \\
(0,00755)\end{array}$ \\
\hline Test d'égalité des coefficients (2) - (1) & $\begin{array}{c}0,156^{\star \star \star} \\
(0,00574)\end{array}$ & $\begin{array}{c}0,182^{\star \star \star} \\
(0,00567)\end{array}$ & $\begin{array}{c}0,288^{\star \star \star} \\
(0,00527)\end{array}$ & $\begin{array}{c}0,186^{\star \star \star} \\
(0,00683)\end{array}$ & $\begin{array}{c}1,438^{\star \star \star} \\
(0,0146)\end{array}$ & $\begin{array}{c}0,156^{\star \star \star} \\
(0,00335)\end{array}$ \\
\hline
\end{tabular}

Lecture : en moyenne, à secteur d'activité, année et effectif donnés, une entreprise implantée à l'étranger a un chiffre d'affaires 52 \% supérieur à celui d'une entreprise domestique. Les écarts-types robustes des coefficients sont entre parenthèses. ${ }^{* * *}$ indique une significativité à $1 \%$, ** à $5 \%$, *à $10 \%$.

Champ : industrie manufacturière, entreprises de plus de 20 salariés, période 2001-2006.

Sources : EAE, LiFi - Calcul des auteurs. 
ou le management. Il apparaît alors que les primoinvestisseurs, dans leur ensemble, connaissent une croissance plus rapide de leur chiffre d'affaires, de leurs effectifs et de leurs exportations que les entreprises ayant choisi de rester en France (cf. tableau $\mathrm{D}$ en annexe 1). L'implantation à l'étranger semble donc récompenser les entreprises les plus performantes, qui renforcent alors leur avantage par rapport aux autres firmes.

\section{Les filiales de groupes s'implantent davantage à l'étranger et en retirent davantage de bénéfices}

Il conviendrait cependant de nuancer ces résultats selon le degré d'indépendance des entreprises. En effet, nous avons vu précédemment qu'il y a davantage de filiales de groupe parmi les multinationales que parmi les exportateurs

\section{Tableau 5}

Primes à l'implantation selon le nombre d'implantation à l'étranger, dans l'industrie manufacturière (période 2001-2006)

\begin{tabular}{|c|c|c|c|c|c|c|}
\hline Statut à l'international & $\begin{array}{c}\text { Productivité } \\
\text { Totale } \\
\text { des Facteurs }\end{array}$ & $\begin{array}{c}\text { Productivité } \\
\text { du travail }\end{array}$ & $\begin{array}{c}\text { Chiffre } \\
\text { d'affaires }\end{array}$ & $\begin{array}{l}\text { Valeur } \\
\text { ajoutée }\end{array}$ & Effectifs & $\begin{array}{l}\text { Salaire } \\
\text { moyen }\end{array}$ \\
\hline \multirow[t]{2}{*}{ Exportation (1) } & $0,0628^{\star \star \star}$ & $0,0928^{\star \star \star}$ & $0,232^{\star \star \star}$ & $0,0946^{\star \star \star}$ & $0,372^{\star \star \star}$ & $0,0817^{\star \star \star}$ \\
\hline & $(0,00455)$ & $(0,00474)$ & $(0,00713)$ & $(0,00485)$ & $(0,00987)$ & $(0,00324)$ \\
\hline \multirow[t]{2}{*}{ Un IDE (2) } & $0,186^{\star \star \star}$ & $0,236^{\star \star \star}$ & $0,486^{\star \star \star}$ & $0,242^{\star \star \star}$ & $1,307^{\star \star \star}$ & $0,200^{\star \star \star}$ \\
\hline & $(0,0144)$ & $(0,0140)$ & $(0,0202)$ & $(0,0145)$ & $(0,0414)$ & $(0,00934)$ \\
\hline \multirow[t]{2}{*}{ Deux IDE (3) } & $0,225^{\star \star \star}$ & $0,276^{\star \star \star}$ & $0,504^{\star \star \star}$ & $0,284^{\star \star \star}$ & $1,721^{\star \star \star}$ & $0,223^{\star \star \star}$ \\
\hline & $(0,0200)$ & $(0,0191)$ & $(0,0260)$ & $(0,0198)$ & $(0,0568)$ & $(0,0121)$ \\
\hline \multirow[t]{2}{*}{ Deux à cinq IDE (4) } & $0,214^{\star \star \star}$ & $0,278^{\star \star \star}$ & $0,547^{\star \star \star}$ & $0,289^{\star \star \star}$ & $2,127^{\star \star \star}$ & $0,261^{\star \star \star}$ \\
\hline & $(0,0244)$ & $(0,0220)$ & $(0,0278)$ & $(0,0230)$ & $(0,0612)$ & $(0,0132)$ \\
\hline \multirow[t]{2}{*}{ Plus de cinq IDE (5) } & $0,312^{\star \star \star}$ & $0,366^{\star \star \star}$ & $0,600^{\star \star \star}$ & $0,380^{\star \star \star}$ & $2,736^{\star \star \star}$ & $0,330^{\star \star \star}$ \\
\hline & $(0,0281)$ & $(0,0236)$ & $(0,0365)$ & $(0,0252)$ & $(0,0669)$ & $(0,0162)$ \\
\hline \multirow[t]{2}{*}{ Test d'égalité des coefficients (2) - (1) } & $0,123^{\star \star \star}$ & $0,143^{\star \star \star}$ & $0,254^{\star \star \star}$ & $0,147^{\star \star \star}$ & $0,935^{\star \star \star}$ & $0,119^{\star \star \star}$ \\
\hline & $(0,00843)$ & $(0,00813)$ & $(0,0117)$ & $(0,00811)$ & $(0,0216)$ & $(0,00572)$ \\
\hline \multirow[t]{2}{*}{ Test d'égalité des coefficients (5) - (2) } & $0,126^{\star \star \star}$ & $0,131^{\star \star \star}$ & $0,114^{\star \star \star}$ & $0,138^{\star \star \star}$ & $1,429^{\star \star \star}$ & $0,130^{\star \star \star}$ \\
\hline & $(0,0157)$ & $(0,0182)$ & $(0,0200)$ & $(0,0154)$ & $(0,0405)$ & $(0,00760)$ \\
\hline
\end{tabular}

Lecture : en moyenne, à secteur d'activité, année et effectif donnés, une entreprise possédant une filiale à l'étranger a un chiffre d'affaires $49 \%$ supérieur à celui d'une entreprise domestique. Les écarts-types robustes des coefficients sont entre parenthèses. ${ }^{* *}$ indique une significativité à $1 \%,{ }^{* *}$ à $5 \%,{ }^{*}$ à $10 \%$.

Champ : industrie manufacturière, entreprises de plus de 20 salariés, période 2001-2006.

Sources : EAE, LiFi - Calcul des auteurs.

Tableau 6

Modélisation de la probabilité de s'implanter à l'étranger dans l'industrie manufacturière

\begin{tabular}{|c|c|c|c|}
\hline \multicolumn{2}{|c|}{ Variable explicative de la probabilité d'investir à l'étranger } & Coefficient & Écart-type \\
\hline \multicolumn{2}{|c|}{ Ln (nombre d'établissements de production) } & $0,090^{*}$ & 0,047 \\
\hline \multicolumn{2}{|c|}{ Ln (Productivité Totale des Facteurs) } & $0,156^{* *}$ & 0,077 \\
\hline \multicolumn{2}{|c|}{$\Delta$ Ln (Productivité Totale des Facteurs) } & $-0,270$ & 0,541 \\
\hline \multicolumn{2}{|l|}{ Ln (chiffre d'affaires) } & $0,212^{\star \star \star}$ & 0,067 \\
\hline \multicolumn{2}{|l|}{$\Delta \operatorname{Ln}$ (chiffre d'affaires) } & $-0,035$ & 0,167 \\
\hline \multicolumn{2}{|l|}{ Ln (effectifs) } & 0,046 & 0,071 \\
\hline \multicolumn{2}{|l|}{$\Delta \operatorname{Ln}($ effectifs) } & $0,423^{\star \star}$ & 0,188 \\
\hline \multicolumn{2}{|c|}{ Exportations/chiffre d'affaires } & $0,614^{\star \star \star}$ & 0,112 \\
\hline \multicolumn{2}{|c|}{ Ln (coût du travail/effectifs) } & $-0,022$ & 0,035 \\
\hline \multicolumn{2}{|c|}{ Ln (immobilisations corporelles) } & 0,181 & 0,132 \\
\hline \multicolumn{2}{|c|}{ Ln (Immobilisations incorporelles/valeur ajoutée) } & $0,054^{\star *}$ & 0,021 \\
\hline \multicolumn{2}{|c|}{ Résultat net/chiffre d'affaires } & $0,818^{\star \star}$ & 0,373 \\
\hline \multirow{4}{*}{$\begin{array}{c}\text { Appartenance à un } \\
\text { groupe (référence : } \\
\text { entreprises } \\
\text { indépendantes) }\end{array}$} & Contrôlée par un groupe étranger & $-0,434^{\star \star *}$ & 0,068 \\
\hline & Contrôlée par un groupe franco-français & $-0,047$ & 0,051 \\
\hline & Contrôlée par un groupe français internationalisé & $0,200^{\star \star}$ & 0,082 \\
\hline & Tête de groupe & $0,331^{\star \star \star}$ & 0,071 \\
\hline
\end{tabular}

Lecture : toutes choses égales par ailleurs, les firmes contrôlées par un groupe étranger ont une probabilité de s'implanter à l'étranger inférieure de 43 points de pourcentage. * indique une significativité à $10 \%$, ** à $5 \%$, *** à $1 \%$.

Champ : industrie manufacturière, entreprises de plus de 20 salariés, période 2001-2006.

Sources : EAE, LiFi - Calcul des auteurs. 
Tableau 7

Primes à l'implantation selon l'appartenance à un groupe, dans l'industrie manufacturière (période 2001-2006)

\begin{tabular}{|c|c|c|c|c|c|c|}
\hline Statut à l'international & $\begin{array}{c}\text { Productivité } \\
\text { Totale des } \\
\text { Facteurs }\end{array}$ & $\begin{array}{c}\text { Productivité } \\
\text { du travail }\end{array}$ & $\begin{array}{c}\text { Chiffre } \\
\text { d'affaires }\end{array}$ & $\begin{array}{c}\text { Valeur } \\
\text { ajoutée }\end{array}$ & Effectifs & $\begin{array}{l}\text { Salaire } \\
\text { moyen }\end{array}$ \\
\hline Multinationale tête de groupe (1) & $\begin{array}{l}0,186^{\star \star \star} \\
(0,0222)\end{array}$ & $\begin{array}{l}0,223^{\star \star \star} \\
(0,0207)\end{array}$ & $\begin{array}{l}0,412^{\star \star \star} \\
(0,0286)\end{array}$ & $\begin{array}{l}0,227^{\star \star \star} \\
(0,0211)\end{array}$ & $\begin{array}{l}1,170^{\star \star \star} \\
(0,0685)\end{array}$ & $\begin{array}{l}0,213^{\star \star \star} \\
(0,0133)\end{array}$ \\
\hline Multinationale contrôlée par un groupe français (2) & $\begin{array}{l}0,215^{\star \star \star} \\
(0,0161)\end{array}$ & $\begin{array}{c}0,265^{\star \star \star} \\
(0,0152)\end{array}$ & $\begin{array}{l}0,516^{\star \star \star} \\
(0,0209)\end{array}$ & $\begin{array}{l}0,272^{\star \star \star} \\
(0,0161)\end{array}$ & $\begin{array}{l}1,894^{\star \star \star} \\
(0,0475)\end{array}$ & $\begin{array}{l}0,220^{\star \star \star} \\
(0,0102)\end{array}$ \\
\hline Multinationale contrôlée par un groupe étranger (3) & $\begin{array}{l}0,245^{\star \star \star} \\
(0,0191)\end{array}$ & $\begin{array}{l}0,321^{\star * \star} \\
(0,0167)\end{array}$ & $\begin{array}{l}0,598^{\star * *} \\
(0,0245)\end{array}$ & $\begin{array}{l}0,329^{\star \star \star} \\
(0,0177)\end{array}$ & $\begin{array}{l}2,089^{\star \star \star} \\
(0,0488)\end{array}$ & $\begin{array}{l}0,277^{\star \star \star} \\
(0,0107)\end{array}$ \\
\hline Test d'égalité des coefficients (3) - (2) & $\begin{array}{c}0,0304^{*} \\
(0,0156)\end{array}$ & $\begin{array}{l}0,0561^{\star \star \star} \\
(0,0161)\end{array}$ & $\begin{array}{l}0,0823^{\star \star *} \\
(0,0143)\end{array}$ & $\begin{array}{l}0,0568^{\star \star \star} \\
(0,0121)\end{array}$ & $\begin{array}{l}0,196^{\star \star \star} \\
(0,0341)\end{array}$ & $\begin{array}{c}0,0566^{\star \star *} \\
(0,00692)\end{array}$ \\
\hline Test d'égalité des coefficients (2) - (1) & $\begin{array}{l}0,0291^{*} \\
(0,0175)\end{array}$ & $\begin{array}{l}0,0417^{\star \star} \\
(0,0172)\end{array}$ & $\begin{array}{l}0,104^{\star \star *} \\
(0,0164)\end{array}$ & $\begin{array}{l}0,0444^{\star * *} \\
(0,0152)\end{array}$ & $\begin{array}{l}0,724^{\star \star *} \\
(0,0370)\end{array}$ & $\begin{array}{c}0,0069 \\
(0,00974)\end{array}$ \\
\hline
\end{tabular}

Lecture : en moyenne, à secteur d'activité, année et effectif donnés, une entreprise multinationale et tête de groupe a un chiffre d'affaires $41 \%$ supérieur à celui d'une entreprise domestique. Les écarts-types robustes des coefficients sont entre parenthèses. ${ }^{* * *}$ indique une significativité à $1 \%,{ }^{* *}$ à $5 \%$, * à $10 \%$.

Champ : industrie manufacturière, entreprises de plus de 20 salariés, période 2001-2006.

Sources : EAE, LiFi - Calcul des auteurs.

ou les firmes domestiques. Ces filiales de groupes se caractérisent d'ailleurs par des primes à l'implantation plus élevées que celles des têtes de groupes (cf. tableau 7) (12).

Ce résultat n'est pas intuitif. En effet, les entreprises têtes de groupe doivent supporter seules le coût de leur implantation à l'étranger, tandis que les filiales peuvent s'appuyer sur les compétences, le réseau et les ressources du groupe auquel elles sont adossées. Ces dernières peuvent également s'épargner le développement de fonctions « support » (marketing, développement de systèmes d'informations, R\&D, etc.), généralement prises en charge par la tête de groupe. Le niveau de productivité requis pour s'implanter à l'étranger devrait donc être plus élevé pour les têtes de groupes que pour les filiales. Selon ce raisonnement, ces dernières devraient donc présenter les primes à l'implantation les plus faibles.

Toutefois, nous observons la hiérarchie inverse, et ce pour deux raisons. Premièrement, les firmes les plus performantes ont une probabilité accrue d'être absorbées par des groupes. Ce phénomène est également connu sous le nom de «cherry picking » : les groupes s'intéressent prioritairement à des sociétés indépendantes à fort potentiel. Or une fois absorbées, ces entre-

12. La variable Ide du modèle initial est maintenant scindée en trois variables Ide tete, Ide cfr et Ide cet qui prennent la valeur 1 si l'entreprise est respectivement tête de groupe, filiale de groupe français ou filiale de groupe étranger ( 0 sinon). Sans surprise, l'introduction d'effets fixes firmes dans la régression conduit à gommer les différences entre têtes et filiales de groupe.

13. Pour approfondir tous ces points, voir Gazaniol et Peltrault (2010). prises ont une probabilité accrue de s'implanter à l'étranger (cf. tableau 6). Autrement dit, l'appartenance à un groupe amplifie le phénomène d'auto-sélection. Deuxièmement, l'implantation à l'étranger s'accompagne d'une amélioration plus marquée des performances pour les filiales que pour les têtes de groupe (cf. tableau $\mathrm{D}$ en annexe 1). En particulier, seuls les primo-investisseurs filiales de groupes connaissent une progression significative de leur productivité (13). Ce constat concerne plus précisément les filiales de groupes français déjà internationalisés : ceci pourrait donc signifier que les effets d'apprentissage liés aux IDE sortants sont essentiellement liés à des effets de réseaux.

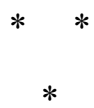

La comparaison exhaustive des performances des multinationales de l'industrie française avec celles des firmes seulement exportatrices et domestiques montre que les primes à l'implantation sont élevées et résistent à l'hétérogénéité de l'échantillon, comme à la prise en compte de caractéristiques non observables des firmes. Elles proviennent tout à la fois d'un effet de sélection et d'une amélioration des performances grâce à l'implantation à l'étranger. $\mathrm{Ce}$ dernier résultat est de nature à tempérer l'inquiétude que font peser les délocalisations sur l'emploi et l'activité en France. Il conviendrait toutefois d'approfondir ces résultats en décrivant plus précisément les mécanismes à l'œuvre derrière les effets d'apprentissage. 


\section{BIBLIOGRAPHIE}

Aw B.Y., Chung S. et Roberts M.J. (2003), «Productivity, output, and failure : a comparison of Taiwanese and Korean manufacturers », Economic Journal, vol. 113, n 491, pp. 485-510.

Barba Navaretti G., Castellani D. et Disdier A. (2010), « How Does Investing in Cheap Labour Countries Affect Performance at Home? », Oxford Economic Papers, vol. 62, n 2, pp. 234-260.

Bellone F., Musso P., Nesta L. et Quéré M. (2006), « Caractéristiques et performances des firmes exportatrices françaises », Revue de l'OFCE, vol. 98, juillet.

Bellone F., Musso P., Nesta L. et Quéré M. (2008), " The U-Shaped Productivity dynamics of French Exporters », Review of World Economics, vol. 144, n ${ }^{\circ}$, pp. 636-659.

Bernard A.B. et Wagner J. (2001), « Export Entry and Exit by German Firms ", Review of World Economics, vol. 137, n 1, pp. 105-123.

Bernard A.B. et Jensen J.B. (1995), « Exporters, Jobs, and Wages in U.S. Manufacturing, 19761987 », Brookings Papers on Economic Activity, Microeconomics, pp. 67-112.

Bernard A.B. et Jensen J.B. (1999), « Exceptional Exporter Performance : Cause, Effect, or Both ?», Journal of International Economics, vol. 47, $\mathrm{n}^{\circ} 1$, pp. 1-26.

Bernard A.B. et Jensen J.B. (2004a), « Exporting and Productivity in the USA », Oxford Review of Economic Policy, vol. 20, n 3, pp. 343-357.

Bernard A.B. et Jensen J.B. (2004b), "Why Some Firms Export? », Review of Economics and Statistics, vol. 86, n 2 , pp. 561-569.

Bernard A.B., Eaton J., Jensen J.B. et Kortum S. (2003), «Plants and Productivity in International Trade ", American Economic Review, vol. 93, $\mathrm{n}^{\circ} 4$, pp. 1268-1290.

Bernard A.B., Jensen J.B., Redding S.J. et Schott P.K. (2007), " Firms in International Trade », Journal of Economic Perspectives, vol. 21, n 3, pp. 105-130.

Bouabdallah O., Gilquin G. et Pincon M.-A. (2008), « L'industrie automobile française face à la mondialisation », la Lettre Trésor-Eco, $\mathrm{n}^{\circ} 43$, septembre.

Brainard L. (1997), « An Empirical Assessment of the Proximity-Concentration Trade-Off between Multinational Sales and Trade ", American Economic Review, vol. 87, pp. 520-544.

Castellani D. et Zanfei A. (2007), « Internationalisation, Innovation and Productivity : how do Firms differ in Italy? », The World Economy, vol. 30, n 1, pp. 156-176.

Clerides S., Lach S. et Tybout J. (1998), « Is Learning-by-Exporting Important ? Micro Dynamic Evidence from Colombia, Mexico, and Morocco ", Quarterly Journal of Economics, vol. 113, n 3, pp. 903-947.

Crozet M., Méjean I. et Zignago S. (2008), « Plus grandes, plus fortes, plus loin ... performances relatives des firmes exportatrices ", dans Fontagné L. et Gaulier G., Performances à l'exportation de la France et de l'Allemagne, Rapport du Conseil d'Analyse Économique, n 81 , pp. 171-186.

De Loecker J. (2007), « Do Exports Generate Higher Productivity ? Evidence from Slovenia », Journal of International Economics, vol. 73, $\mathrm{n}^{\circ} 1$, pp. 69-98.

Doms M. et Jensen J.B. (1998), « Comparing Wages, Skills, and Productivity between Domestically and Foreign-Owned Manufacturing Establishments in the United States » in Baldwin R.E., Lipsey R.E. and Richardson J.D. (eds.), Geography and Ownership as Bases for Economic Accounting, Chicago, University of Chicago Press.

Dovis M. (2007), « Ouverture aux échanges internationaux et Productivité Totale des Facteur : une analyse au niveau des firmes », Thèse de doctorat, Université de la Méditerranée Aix-Marseille II.

Eaton J., Kortum S. et Kramarz F. (2004), « Dissecting Trade : Firms, Industries, and Export Destinations ", American Economic Review, Papers and Proceedings, vol. 93, pp. 150-154.

Fontagné L. et Gaulier G. (2008), Performances à l'exportation de la France et de l'Allemagne, Rapport du Conseil d'Analyse Économique $\mathrm{n}^{\circ} 81$. 
Gazaniol A. et Peltrault F. (2010), « La première implantation à l'étranger des entreprises françaises : un impact différencié selon l'appartenance à un groupe », dans Fontagné L. et Toubal F. Investissement Direct Étranger et performances des entreprises, Rapport du Conseil d'Analyse Économique, pp. 117-142.

Girma S., Greenaway D. et Wakelin K. (2001), «Who Benefits from Foreign Direct Investment in the UK? », Scottish Journal of Political Economy, vol. 48, n², pp. 119-133.

Girma S., Greenaway D. et Kneller R. (2003), «Export Market Exit and Performance. Dynamics : A Causality Analysis of Matched Firms ", Economics Letters, vol. 80, $\mathrm{n}^{\circ}$ 2, pp. 181-187.

Girma S., Greenaway D. et Kneller R. (2004), « Does Exporting Increase Productivity ? : A Microeconometric Analysis of Matched Firms ", Review of International Economics, vol. 12, $\mathrm{n}^{\circ} 5$, pp. 855-866.

Globerman S., Ries J. et Vertinsky I. (1994), « The Economic Performance of Foreign Affiliates in Canada ", Canadian Journal of Economics, vol. $27, \mathrm{n}^{\circ} 1$, pp. 143-156.

Gourlaouen Y. (2004), « La chimie de base à l'heure de l'internationalisation "), Sessi, Le 4 pages des statistiques industrielles, ${ }^{\circ} 194$.

Greenaway D. et Kneller R. (2007), « Firm Heterogeneity, Exporting and Foreign Direct Investment ", The Economic Journal, vol. 117, pp. 134-161.

Hall B. et Mairesse J. (1995), « Exploring the Relationship between R\&D and Productivity in French Manufacturing Firms ", Journal of Econometrics, vol. 65, $\mathrm{n}^{\circ} 1$, pp. 263-293.

Head K. et Ries J. (2003), « Heterogeneity and the FDI versus Export Decision of Japanese Manufacturers "), Journal of the Japanese and International Economies, vol. 17, $\mathrm{n}^{\circ} 4$, pp. 448467.

Helpman E. et Krugman P. (1985), Market Structure and Foreign Trade, MIT Press.

Helpman E., Melitz M. et Yeaple S. (2004), " Export versus FDI with heterogeneous Firms », The American Economic Review, vol. 94, $\mathrm{n}^{\circ} 1$, pp. 310-316.

Hijzen A., Jean S. et Mayer T. (2009), « The Effects at Home of Initiating Production Abroad :
Evidence from French Matched Firms », Cepii working Paper, $\mathrm{n}{ }^{\circ}$ 2009-39.

Isgep (2008), « Understanding Cross-Country Differences in Exporter Premia : Comparable Evidence for 14 Countries », Review of World Economics, vol. 144, n ${ }^{\circ}$, pp. 596-635.

Isgut A. et Fernandes A. (2007), « Learning-byExporting Effects : Are They for Real ? ", MPRA Paper, $\mathrm{n}^{\circ} 3121$, University Library of Munich, Germany.

Kleinert J. et Toubal F. (2007), The Impact of Locating Production Abroad on Activities at Home : Evidence from German Firm-Level Data, University of Tübingen Mimeo.

Koenker R. et Basset G. (1978), « Regression Quantiles », Econometrica, vol. 46, $\mathrm{n}^{\circ} 1$, pp. 33-50.

Koenker R. et Hallock K.F. (2001), « Quantile Regression ", Journal of Economic Perspectives, vol. $15, \mathrm{n}^{\circ} 4$, pp. 143-156.

Levinsohn J. et Petrin A. (2003), « Estimating Production Functions using Inputs to Control for Unobservables », Review of Economic Studies, vol. $70, \mathrm{n}^{\circ} 2$, pp. 317-341.

Lileeva A. et Trefler D. (2007), « Improved Access to Foreign Markets Raises Plant-Level Productivity for Some Plants », NBER Working Paper, $\mathrm{n}^{\circ} 13297$.

Mayer T. et Ottaviano G.I.P. (2007), The Happy Few : the Internationalisation of European Firms, Bruegel-CEPR EFIM2007 Report. Bruegel Blueprint Series.

Melitz M.J. (2003), « The Impact of Trade on Intra-industry Reallocations and Aggregate Industry Productivity »Econometrica, vol. 71, $\mathrm{n}^{\circ} 6$, pp. 1695-1725.

Nefussi B. (2007), « Les groupes absorbent des sociétés à fort potentiel », Insee Première, $\mathrm{n}^{\circ} 1144$.

Olley S. et Pakes A. (1996), « The Dynamics of Productivity in the Telecommunications Equipment Industry », Econometrica, vol. 64, n 6, pp. 1263-98.

Pliquet E. et Riedinger N. (2008), « Les implantations à l'étranger des entreprises industrielles françaises ", Sessi, Le 4 pages des statistiques industrielles, $\mathrm{n}^{\circ} 246$, mai. 
Siroen J.-M. (1985), Les effets d'entraînement par les exportations, Thèse d'État, Université Paris-Dauphine.

Tomiura E. (2007), « Foreign Outsourcing, Exporting, and FDI : A Productivity Comparison at the Firm Level ", Journal of International Economics, vol. 72, n ${ }^{\circ}$, pp. 113-127.

Van Biesebroeck J. (2005), « Exporting Raises Productivity in sub-SaharanAfrican Manufacturing Firms », Journal of International Economics, vol. $67, \mathrm{n}^{\circ} 2$, pp. 373-391.

Van Biesebroeck J. (2008), « The Sensitivity of Productivity Estimates : Revisiting Three Important Productivity Debates », Journal of Business and Economic Statistics, vol. 26, $\mathrm{n}^{\circ} 3$, pp. 321-238.

Wagner J. (2002), " The Causal Effects of Exports on Firm Size and Labor Productivity :
First Evidence from a Matching Approach », Economics Letters, vol. 77, n² 2, pp. 287-292.

Wagner J. (2005), « Exports, Foreign Direct Investment and Productivity : Evidence from German Firm Level Data ", HWWA Discussion Paper, $\mathrm{n}^{\circ} 218$.

Wagner J. (2007), « Exports and Productivity : A Survey of the Evidence from Firm-Level Data », The World Economy, vol. 30, n ${ }^{\circ}$, pp. 60-82.

Yasar M., Nelson C.H. et Rejesus R. (2006), « Productivity and Exporting Status of Manufacturing Firms : Evidence from Quantile Regressions ", Review of World Economics, vol. $142 n^{\circ} 4$, pp. 675-694.

Yasar M., Morrison P. et Catherine J. (2007), « International Linkages and Productivity at the Plant Level : Foreign Direct Investment, Exports, Imports and Licensing », Journal of International Economics, vol. 71, n 2 , pp. 373-388. 


\section{STATISTIQUES COMPLÉMENTAIRES}

Tableau A

Performances moyennes des entreprises industrielles selon leur statut à l'international et leur secteur d'activité en 2006

\begin{tabular}{|c|c|c|c|c|c|c|c|c|}
\hline $\begin{array}{c}\text { Secteur d'activité } \\
\text { (NES à } \\
36 \text { niveaux) }\end{array}$ & Statut à l'international & $\begin{array}{c}\text { Nombre } \\
\text { d'entreprises }\end{array}$ & $\begin{array}{c}\text { Chiffre } \\
\text { d'affaires } \\
\text { en milliers } \\
\text { d'euros }\end{array}$ & $\begin{array}{l}\text { Valeur } \\
\text { ajoutée } \\
\text { en milliers } \\
\text { d'euros }\end{array}$ & Effectifs & $\begin{array}{l}\text { Ratio } \\
\text { valeur } \\
\text { ajoutée / } \\
\text { effectif }\end{array}$ & $\begin{array}{c}\text { Salaire } \\
\text { mensuel } \\
\text { brut en } \\
\text { milliers } \\
\text { d'euros }\end{array}$ & $\begin{array}{c}\text { Intensité } \\
\text { d'exportation } \\
\text { en } \%\end{array}$ \\
\hline \multirow{3}{*}{$\begin{array}{l}\text { Habillement et } \\
\text { Cuir }\end{array}$} & Domestique & 117 & 2324 & 1364 & 48 & 30 & 1,56 & 0 \\
\hline & Exportatrice & 448 & 9548 & 3142 & 73 & 47 & 2 & 23 \\
\hline & Implantée à l'étranger & 67 & 52054 & 16862 & 286 & 59 & 2,23 & 36 \\
\hline \multirow{3}{*}{$\begin{array}{c}\text { Édition, } \\
\text { imprimerie } \\
\text { et reproduction }\end{array}$} & Domestique & 442 & 8005 & 3659 & 66 & 52 & 2,32 & 0 \\
\hline & Exportatrice & 766 & 14772 & 5409 & 87 & 58 & 2,48 & 8 \\
\hline & Implantée à l'étranger & 35 & 98925 & 29695 & 361 & 80 & 3,13 & 17 \\
\hline \multirow{3}{*}{$\begin{array}{l}\text { Pharmacie, } \\
\text { Parfums et } \\
\text { Entretien }\end{array}$} & Domestique & 38 & 30698 & 10227 & 121 & 81 & 2,64 & 0 \\
\hline & Exportatrice & 319 & 65885 & 20468 & 202 & 80 & 2,62 & 29 \\
\hline & Implantée à l'étranger & 74 & 319671 & 101124 & 847 & 112 & 3,23 & 44 \\
\hline \multirow{3}{*}{$\begin{array}{l}\text { Équipements du } \\
\text { Foyer }\end{array}$} & Domestique & 179 & 6754 & 2236 & 55 & 47 & 1,93 & 0 \\
\hline & Exportatrice & 726 & 19478 & 6374 & 119 & 54 & 2,06 & 21 \\
\hline & Implantée à l'étranger & 74 & 70381 & 22290 & 388 & 61 & 2,44 & 36 \\
\hline \multirow{3}{*}{$\begin{array}{l}\text { Industrie } \\
\text { Automobile }\end{array}$} & Domestique & 53 & 5552 & 1934 & 41 & 49 & 1,96 & 0 \\
\hline & Exportatrice & 349 & 47966 & 14602 & 226 & 57 & 2,07 & 24 \\
\hline & Implantée à l'étranger & 25 & 447069 & 94651 & 1268 & 67 & 2,22 & 43 \\
\hline \multirow{3}{*}{$\begin{array}{l}\text { Construction } \\
\text { navale, } \\
\text { aéronautique et } \\
\text { ferroviaire }\end{array}$} & Domestique & 43 & 4140 & 1129 & 58 & 19 & 1,74 & 0 \\
\hline & Exportatrice & 169 & 43013 & 9195 & 258 & 26 & 1,96 & 32 \\
\hline & Implantée à l'étranger & 23 & 326112 & 60817 & 1577 & 29 & 2,1 & 52 \\
\hline \multirow{3}{*}{$\begin{array}{l}\text { Équipements } \\
\text { mécaniques }\end{array}$} & Domestique & 892 & 5929 & 2284 & 47 & 49 & 2 & 0 \\
\hline & Exportatrice & 1940 & 15206 & 5145 & 90 & 54 & 2,15 & 22 \\
\hline & Implantée à l'étranger & 184 & 82986 & 30905 & 455 & 63 & 2,43 & 47 \\
\hline \multirow{3}{*}{$\begin{array}{l}\text { Équipements } \\
\text { électriques / } \\
\text { électroniques }\end{array}$} & Domestique & 200 & 6954 & 5117 & 52 & 102 & 3,27 & 0 \\
\hline & Exportatrice & 563 & 25081 & 12433 & 102 & 117 & 3,57 & 27 \\
\hline & Implantée à l'étranger & 91 & 254740 & 101882 & 689 & 143 & 4,24 & 48 \\
\hline \multirow{3}{*}{ Produits Minéraux } & Domestique & 553 & 10714 & 3425 & 59 & 60 & 1,7 & 0 \\
\hline & Exportatrice & 500 & 17059 & 6260 & 110 & 51 & 1,74 & 20 \\
\hline & Implantée à l'étranger & 50 & 156081 & 57102 & 870 & 63 & 2,06 & 34 \\
\hline \multirow{3}{*}{ Textile } & Domestique & 91 & 3671 & 2135 & 52 & 40 & 1,72 & 0 \\
\hline & Exportatrice & 555 & 10682 & 3649 & 76 & 48 & 1,94 & 29 \\
\hline & Implantée à l'étranger & 34 & 37550 & 9955 & 210 & 49 & 2,1 & 48 \\
\hline \multirow{3}{*}{ Bois et Papier } & Domestique & 364 & 6638 & 2521 & 54 & 47 & 1,82 & 0 \\
\hline & Exportatrice & 644 & 16571 & 5211 & 99 & 51 & 1,95 & 17 \\
\hline & Implantée à l'étranger & 38 & 118557 & 32123 & 486 & 61 & 2,33 & 35 \\
\hline \multirow{3}{*}{$\begin{array}{c}\text { Chimie, } \\
\text { caoutchouc et } \\
\text { plastiques }\end{array}$} & Domestique & 283 & 12353 & 4774 & 71 & 63 & 1,78 & 0 \\
\hline & Exportatrice & 1362 & 20096 & 8453 & 115 & 71 & 1,96 & 24 \\
\hline & Implantée à l'étranger & 171 & 109275 & 45219 & 505 & 89 & 2,25 & 40 \\
\hline \multirow{3}{*}{ Métallurgie } & Domestique & 709 & 4474 & 1763 & 49 & 37 & 1,62 & 0 \\
\hline & Exportatrice & 2266 & 9882 & 3252 & 83 & 40 & 1,68 & 20 \\
\hline & Implantée à l'étranger & 107 & 92277 & 26701 & 552 & 48 & 1,79 & 38 \\
\hline \multirow{3}{*}{$\begin{array}{l}\text { Composants } \\
\text { électriques/ } \\
\text { électroniques }\end{array}$} & Domestique & 106 & 7706 & 3695 & 59 & 63 & 2,06 & 0 \\
\hline & Exportatrice & 484 & 33383 & 14013 & 166 & 78 & 2,37 & 25 \\
\hline & Implantée à l'étranger & 73 & 202845 & 64966 & 782 & 83 & 2,81 & 49 \\
\hline
\end{tabular}

Lecture : en 2006, les entreprises exportatrices dans la métallurgie ont un effectif moyen de 83 salariés et une intensité d'exportation de $20 \%$. Toutes les valeurs sont des moyennes.

Champ : industrie manufacturière, entreprises de plus de 20 salariés.

Sources : EAE, LiFi - Calcul des auteurs. 
Tableau B

Primes de performances en intégrant des effets fixes firmes

\begin{tabular}{|c|c|c|c|c|c|c|c|}
\hline \multirow[b]{2}{*}{ Statut à l'international } & \multirow[b]{2}{*}{$\begin{array}{c}\text { Nombre } \\
\text { d'observations }\end{array}$} & \multicolumn{6}{|c|}{ Primes de performances } \\
\hline & & $\begin{array}{c}\text { Productivité } \\
\text { Totale des } \\
\text { Facteurs }\end{array}$ & $\begin{array}{c}\text { Productivité } \\
\text { du travail }\end{array}$ & $\begin{array}{c}\text { Chiffre } \\
\text { d'affaires }\end{array}$ & $\begin{array}{c}\text { Valeur } \\
\text { ajoutée }\end{array}$ & Effectifs & Salaires \\
\hline Exportation (1) & 71088 & $\begin{array}{l}0,0155^{\star \star \star} \\
(0,0045)\end{array}$ & $\begin{array}{l}0,0159^{\star \star \star} \\
(0,0045)\end{array}$ & $\begin{array}{l}0,0466^{\star \star \star} \\
(0,0042)\end{array}$ & $\begin{array}{l}0,0214^{\star \star \star} \\
(0,0045)\end{array}$ & $\begin{array}{l}0,0276^{\star \star \star} \\
(0,0035)\end{array}$ & $\begin{array}{l}0,0142^{\star \star \star} \\
(0,0029)\end{array}$ \\
\hline Implantation (2) & 6370 & $\begin{array}{l}0,0464^{\star \star \star} \\
(0,0133)\end{array}$ & $\begin{array}{l}0,0382^{\star \star *} \\
(0,0131)\end{array}$ & $\begin{array}{l}0,0680^{\star * *} \\
(0,0082)\end{array}$ & $\begin{array}{l}0,0560^{\star * *} \\
(0,0131)\end{array}$ & $\begin{array}{l}0,0890^{\star * *} \\
(0,0118)\end{array}$ & $\begin{array}{l}0,0359^{* * *} \\
(0,0062)\end{array}$ \\
\hline Test d'égalité des coefficients (2) - (1) & - & $\begin{array}{l}0,0309^{\star \star \star} \\
(0,0113)\end{array}$ & $\begin{array}{l}0,0223^{\star \star} \\
(0,0104)\end{array}$ & $\begin{array}{l}0,0213^{\star \star \star} \\
(0,0077)\end{array}$ & $\begin{array}{l}0,0346^{\star *} \\
(0,0135)\end{array}$ & $\begin{array}{l}0,0615^{\star \star \star} \\
(0,0112)\end{array}$ & $\begin{array}{l}0,0218^{\star \star \star} \\
(0,0050)\end{array}$ \\
\hline Un IDE (3) & 2710 & $\begin{array}{l}0,0339^{\star *} \\
(0,0132)\end{array}$ & $\begin{array}{l}0,0286^{\star *} \\
(0,0131)\end{array}$ & $\begin{array}{l}0,0586^{\star \star \star} \\
(0,0084)\end{array}$ & $\begin{array}{l}0,0423^{\star \star *} \\
(0,0131)\end{array}$ & $\begin{array}{l}0,0683^{\star \star \star} \\
(0,0110)\end{array}$ & $\begin{array}{l}0,0276^{\star \star \star} \\
(0,0063)\end{array}$ \\
\hline Deux IDE (4) & 1153 & $\begin{array}{l}0,0742^{\star \star \star} \\
(0,0200)\end{array}$ & $\begin{array}{l}0,0608^{\star \star *} \\
(0,0195)\end{array}$ & $\begin{array}{l}0,0871^{\star \star \star} \\
(0,0123)\end{array}$ & $\begin{array}{l}0,0852^{\star \star \star} \\
(0,0197)\end{array}$ & $\begin{array}{l}0,1220^{\star \star \star} \\
(0,0190)\end{array}$ & $\begin{array}{l}0,0503^{\star * *} \\
(0,0094)\end{array}$ \\
\hline Deux à cinq IDE (5) & 1381 & $\begin{array}{l}0,0737^{\star \star} \\
(0,0321)\end{array}$ & $\begin{array}{c}0,0566^{*} \\
(0,0315)\end{array}$ & $\begin{array}{l}0,0953^{\star \star \star} \\
(0,0135)\end{array}$ & $\begin{array}{l}0,0882^{\star \star \star} \\
(0,0316)\end{array}$ & $\begin{array}{l}0,1580^{\star \star \star} \\
(0,0221)\end{array}$ & $\begin{array}{l}0,0673^{\star \star \star} \\
(0,0108)\end{array}$ \\
\hline Cinq IDE et plus (6) & 1126 & $\begin{array}{l}0,1220^{\star \star \star} \\
(0,0401)\end{array}$ & $\begin{array}{l}0,0941^{\star *} \\
(0,0384)\end{array}$ & $\begin{array}{l}0,1090^{\star \star \star} \\
(0,0179)\end{array}$ & $\begin{array}{l}0,1380^{\star * *} \\
(0,0392)\end{array}$ & $\begin{array}{l}0,2180^{\star * *} \\
(0,0319)\end{array}$ & $\begin{array}{l}0,0663^{\star * *} \\
(0,0172)\end{array}$ \\
\hline Test d'égalité des coefficients (3) - (1) & - & $\begin{array}{c}0,0183 \\
(0,0140)\end{array}$ & $\begin{array}{c}0,0127 \\
(0,0138)\end{array}$ & $\begin{array}{c}0,0119^{*} \\
(0,0068)\end{array}$ & $\begin{array}{l}0,0208^{\star *} \\
(0,0105)\end{array}$ & $\begin{array}{l}0,0407^{\star \star \star} \\
(0,0108)\end{array}$ & $\begin{array}{l}0,0134^{\star *} \\
(0,0055)\end{array}$ \\
\hline Test d'égalité des coefficients (6) - (3) & - & $\begin{array}{l}0,0880^{\star \star} \\
(0,0437)\end{array}$ & $\begin{array}{c}0,0655 \\
(0,0409)\end{array}$ & $\begin{array}{l}0,0505^{\star \star \star} \\
(0,0174)\end{array}$ & $\begin{array}{l}0,0955^{\star \star} \\
(0,0373)\end{array}$ & $\begin{array}{l}0,1500^{\star \star \star} \\
(0,0308)\end{array}$ & $\begin{array}{l}0,0387^{\star *} \\
(0,0169)\end{array}$ \\
\hline Multinationales têtes de groupe (7) & 1323 & $\begin{array}{l}0,0661^{\star \star \star} \\
(0,0212)\end{array}$ & $\begin{array}{l}0,0627^{\text {**}} \\
(0,0206)\end{array}$ & $\begin{array}{l}0,0655^{\star \star \star} \\
(0,0132)\end{array}$ & $\begin{array}{l}0,0770^{\star \star \star} \\
(0,0210)\end{array}$ & $\begin{array}{l}0,0716^{\star \star \star} \\
(0,0172)\end{array}$ & $\begin{array}{l}0,0411^{\star * *} \\
(0,0115)\end{array}$ \\
\hline $\begin{array}{l}\text { Multinationales contrôlées par un } \\
\text { groupe français }(8)\end{array}$ & 2929 & $\begin{array}{l}0,0405^{\star *} \\
(0,0174)\end{array}$ & $\begin{array}{l}0,0305^{\star} \\
(0,0173)\end{array}$ & $\begin{array}{l}0,0668^{\star \star *} \\
(0,0096)\end{array}$ & $\begin{array}{l}0,0487^{\star * \star} \\
(0,0172)\end{array}$ & $\begin{array}{l}0,0915^{\star \star \star} \\
(0,0135)\end{array}$ & $\begin{array}{l}0,0323^{\star \star \star} \\
(0,0076)\end{array}$ \\
\hline $\begin{array}{l}\text { Multinationales contrôlées par un } \\
\text { groupe étranger (9) }\end{array}$ & 2118 & $\begin{array}{l}0,0418^{\star *} \\
(0,0195)\end{array}$ & $\begin{array}{l}0,0333^{*} \\
(0,0189)\end{array}$ & $\begin{array}{l}0,0716^{\star \star \star} \\
(0,0115)\end{array}$ & $\begin{array}{l}0,0528^{\star \star \star} \\
(0,0191)\end{array}$ & $\begin{array}{l}0,0973^{\star \star \star} \\
(0,0169)\end{array}$ & $\begin{array}{l}0,0381^{\star \star \star} \\
(0,0089)\end{array}$ \\
\hline Test d'égalité des coefficients (9) - (8) & - & $\begin{array}{l}0,0013 \\
(0,0196)\end{array}$ & $\begin{array}{l}0,0029 \\
(0,0216)\end{array}$ & $\begin{array}{l}0,0048 \\
(0,0107)\end{array}$ & $\begin{array}{l}0,0040 \\
(0,0184)\end{array}$ & $\begin{array}{c}0,0058 \\
(0,0138)\end{array}$ & $\begin{array}{r}0,0059 \\
(0,0109)\end{array}$ \\
\hline Test d'égalité des coefficients (8) - (7) & - & $\begin{array}{r}-0,0256 \\
(0,0259)\end{array}$ & $\begin{array}{r}-0,0323 \\
(0,0303)\end{array}$ & $\begin{array}{l}0,00124 \\
(0,0151)\end{array}$ & $\begin{array}{l}-0,0283 \\
(0,0268)\end{array}$ & $\begin{array}{r}0,0199 \\
(0,0153)\end{array}$ & $\begin{array}{l}-0,0089 \\
(0,0105)\end{array}$ \\
\hline
\end{tabular}

Lecture : en moyenne, à secteur d'activité, année et effectif donnés, une entreprise implantée à l'étranger a un chiffre d'affaires $4 \%$ supérieur à celui d'une entreprise domestique. Les écarts-types robustes des coefficients sont entre parenthèses. ${ }^{* * *}$ indique une significativité à $1 \%,{ }^{* *}$ à $5 \%,{ }^{*}$ à $10 \%$.

Champ : industrie manufacturière, entreprises de plus de 20 salariés, période 2001-2006.

Sources : EAE, LiFi - Calcul des auteurs. 
Tableau C

Primes à l'exportation et à l'implantation calculée par la régression quantile, dans l'industrie manufacturière (période 2001 - 2006)

\begin{tabular}{|c|c|c|c|c|c|c|c|}
\hline \multirow{2}{*}{ Variable } & \multirow{2}{*}{ Prime } & \multirow{2}{*}{$\begin{array}{c}\text { Estimation } \\
\text { par les MCO }\end{array}$} & \multicolumn{5}{|c|}{ Estimation par la régression quantile } \\
\hline & & & 0,10 & 0,25 & 0,50 & 0,75 & 0,90 \\
\hline \multirow{4}{*}{$\begin{array}{c}\text { Productivité } \\
\text { Totale } \\
\text { des Facteurs }\end{array}$} & Exportation & $0,0621^{*}$ & $0,0382^{*}$ & $0,0516^{*}$ & $0,0598^{*}$ & $0,0768^{*}$ & $0,0915^{\prime}$ \\
\hline & & $(0,0046)$ & $(0,0051)$ & $(0,0034)$ & $(0,0031)$ & $(0,0039)$ & $(0,0051)$ \\
\hline & Implantation & $0,218^{*}$ & $0,1571^{*}$ & $0,1933^{*}$ & $0,2257^{*}$ & $0,2529^{*}$ & 0,2694 \\
\hline & & $(0,0121)$ & $(0,0111)$ & $(0,0083)$ & $(0,0071)$ & $(0,0064)$ & $(0,0102)$ \\
\hline \multirow{4}{*}{$\begin{array}{c}\text { Productivité } \\
\text { du travail }\end{array}$} & Exportation & $0,0927^{*}$ & $0,0703^{*}$ & $0,0858^{*}$ & $0,0918^{\star}$ & $0,1088^{\star}$ & 0,1183 \\
\hline & & $(0,0047)$ & $(0,0049)$ & $(0,0028)$ & $(0,0036)$ & $(0,0030)$ & $(0,0036)$ \\
\hline & Implantation & $0,275^{\star}$ & $0,2342^{*}$ & $0,2727^{*}$ & $0,2953^{*}$ & $0,3040^{\star}$ & $0,2842^{\prime}$ \\
\hline & & $(0,0107)$ & $(0,0127)$ & $(0,0061)$ & $(0,0090)$ & $(0,0082)$ & $(0,0077)$ \\
\hline \multirow{4}{*}{$\begin{array}{c}\text { Chiffre } \\
\text { d'affaires }\end{array}$} & Exportation & $0,231^{\star}$ & $0,2021^{*}$ & $0,1848^{*}$ & $0,1851^{*}$ & $0,2326^{\star}$ & $0,2753^{\prime}$ \\
\hline & & $(0,0071)$ & $(0,0039)$ & $(0,0044)$ & $(0,0031)$ & $(0,0035)$ & $(0,0049)$ \\
\hline & Implantation & $0,519^{\star}$ & $0,4306^{*}$ & $0,4060^{*}$ & $0,4326^{\star}$ & $0,4814^{*}$ & $0,5661^{\star}$ \\
\hline & & $(0,0165)$ & $(0,0130)$ & $(0,0118)$ & $(0,009)$ & $(0,0122)$ & $(0,0125)$ \\
\hline \multirow{4}{*}{$\begin{array}{l}\text { Valeur } \\
\text { ajoutée }\end{array}$} & Exportation & $0,0937^{*}$ & $0,0723^{*}$ & $0,0869^{*}$ & $0,0906^{\star}$ & $0,1073^{*}$ & $0,1202^{\prime}$ \\
\hline & & $(0,0049)$ & $(0,0051)$ & $(0,0035)$ & $(0,0044)$ & $(0,0041)$ & $(0,0056)$ \\
\hline & Implantation & $0,280^{*}$ & $0,2499^{*}$ & $0,2778^{\star}$ & $0,2877^{*}$ & $0,2979^{*}$ & $0,2958^{\star}$ \\
\hline & & $(0,0119)$ & $(0,0134)$ & $(0,0091)$ & $(0,0067)$ & $(0,0084)$ & $(0,0099)$ \\
\hline \multirow{4}{*}{ Effectifs } & Exportation & $0,371^{*}$ & $0,0870^{*}$ & $0,1719^{*}$ & $0,2749^{*}$ & $0,5714^{*}$ & $0,7771^{\star}$ \\
\hline & & $(0,0099)$ & $(0,0011)$ & $(0,0044)$ & $(0,0067)$ & $(0,0089)$ & $(0,0129)$ \\
\hline & Implantation & $1,809^{*}$ & $0,7607^{\star}$ & $1,4282^{*}$ & $1,9107^{*}$ & $2,3461^{*}$ & $2,5319^{\star}$ \\
\hline & & $(0,0337)$ & $(0,0209)$ & $(0,0231)$ & $(0,0162)$ & $(0,0256)$ & $(0,0295)$ \\
\hline \multirow{4}{*}{$\begin{array}{l}\text { Salaire } \\
\text { moyen }\end{array}$} & Exportation & $0,0808^{*}$ & $0,0803^{*}$ & $0,0756^{*}$ & $0,0755^{\star}$ & $0,0781^{*}$ & $0,0831^{x}$ \\
\hline & & $(0,0032)$ & $(0,0034)$ & $(0,0028)$ & $(0,0024)$ & $(0,0020)$ & $(0,0033)$ \\
\hline & Implantation & $0,237^{*}$ & $0,2426^{*}$ & $0,2285^{\star}$ & $0,2187^{*}$ & $0,2204^{*}$ & $0,2408^{\star}$ \\
\hline & & $(0,0076)$ & $(0,0061)$ & $(0,0041)$ & $(0,0053)$ & $(0,0050)$ & $(0,0085)$ \\
\hline
\end{tabular}

Lecture : les écarts-types sont entre parenthèses. * signifie que le coefficient est significatif à $1 \%$, ** à $5 \%$, *** à $10 \%$. Champ : industrie manufacturière, entreprises de plus de 20 salariés.

Sources : EAE, LiFi - Calcul des auteurs.

Tableau D

Impact de l'implantation à l'étranger sur les performances des entreprises, mesuré par l'estimateur " double différence »

\begin{tabular}{|c|c|c|c|c|c|c|c|c|c|}
\hline \multirow[b]{2}{*}{ VARIABLE } & \multirow[b]{2}{*}{ PÉRIODE } & \multicolumn{8}{|c|}{ DEGRÉ D'INDEPENDANCE DES PRIMO-INVESTISSEURS } \\
\hline & & $\begin{array}{c}\text { Tous } \\
\text { primo- } \\
\text { investis- } \\
\text { seurs }\end{array}$ & $\begin{array}{l}\text { Têtes de } \\
\text { groupe }\end{array}$ & $\begin{array}{l}\text { Indépen- } \\
\text { dants }\end{array}$ & $\begin{array}{l}\text { Filiales } \\
\text { de groupe }\end{array}$ & $\begin{array}{l}\text { Filiales de } \\
\text { groupes } \\
\text { étrangers }\end{array}$ & $\begin{array}{l}\text { Filiales de } \\
\text { groupes } \\
\text { français }\end{array}$ & $\begin{array}{l}\text { Filiales de } \\
\text { groupes } \\
\text { franco- } \\
\text { français }\end{array}$ & $\begin{array}{c}\text { Filiales de } \\
\text { groupes } \\
\text { français } \\
\text { internatio- } \\
\text { nalisées }\end{array}$ \\
\hline \multirow{2}{*}{ Effectifs } & $\mathrm{T}$ & $0,033^{\star \star}$ & 0,0215 & 0,0071 & $0,0446^{\star \star}$ & $-0,0019$ & $0,0682^{\star \star}$ & $0,0555^{\star}$ & 0,1103 \\
\hline & $\mathrm{T}+3$ & $0,0657^{\star \star *}$ & $0,1104^{*}$ & 0,013 & $0,0719^{\star \star}$ & 0,036 & $0,0902^{\star \star \star}$ & $0,085^{\star \star}$ & 0,1076 \\
\hline \multirow{2}{*}{$\begin{array}{c}\text { Chiffre } \\
\text { d'affaires }\end{array}$} & $\mathrm{T}$ & 0,0299 & $-0,0018$ & $0,061^{\star *}$ & 0,0273 & $-0,0038$ & 0,043 & 0,0267 & 0,0975 \\
\hline & $\mathrm{T}+3$ & $0,0803^{\star \star \star}$ & 0,0828 & $0,1221^{*}$ & $0,0656^{\star \star}$ & 0,0485 & 0,0743 & $0,0436^{\star \star}$ & $0,1766^{\star}$ \\
\hline \multirow{2}{*}{$\begin{array}{l}\text { Valeur } \\
\text { ajoutée }\end{array}$} & $\mathrm{T}$ & 0,0381 & $-0,0442$ & 0,0527 & 0,0542 & $-0,0402$ & $0,1021^{\star \star}$ & $0,0883^{*}$ & 0,148 \\
\hline & $T+3$ & $0,093^{\star \star}$ & 0,0706 & 0,0372 & $0,1176^{\star \star}$ & 0,071 & $0,1412^{\star \star}$ & 0,1048 & $0,2626^{\star \star}$ \\
\hline \multirow{2}{*}{$\begin{array}{c}\text { Intensité } \\
\text { d'exportation }\end{array}$} & $\mathrm{T}$ & $0,0161^{\star \star}$ & - 0,0098 & $0,0272^{*}$ & $0,019^{\star \star}$ & 0,0264 & 0,0152 & 0,0189 & 0,0029 \\
\hline & $T+3$ & $0,0332^{\star *}$ & 0,0181 & 0,0064 & $0,0441^{\star \star \star}$ & 0,0236 & $0,0545^{\star \star \star}$ & $0,0729^{\star \star \star}$ & $-0,0069$ \\
\hline \multirow{2}{*}{$\begin{array}{c}\text { Productivité Totale } \\
\text { des Facteurs }\end{array}$} & $\mathrm{T}$ & 0,0144 & $-0,0407$ & 0,0483 & 0,0171 & $-0,0463$ & 0,0493 & 0,0487 & 0,0515 \\
\hline & $T+2$ & 0,0383 & 0,0002 & 0,0041 & $0,0596^{\star \star \star}$ & $-0,0341$ & $0,1071^{\star *}$ & $0,0834^{*}$ & $0,1863^{* *}$ \\
\hline
\end{tabular}

Lecture : en $T+3$, les primo-investisseurs têtes de groupe ont un effectif supérieur de $11 \%$ par rapport au scénario où elles auraient reporté leur investissement. Pour la Productivité Totale des Facteurs, nous ne reportons que le coefficient en T-2, par manque d'observations en $T$-3. * indique une significativité à $10 \%$, ** à $5 \%$, et ${ }^{* * *}$ à $1 \%$.

Champ : industrie manufacturière, entreprises de plus de 20 salariés.

Sources : EAE Industrie, LiFi - tableau tiré de Gazaniol et Peltrault (2010). 


\section{LES MESURES DE LA PRODUCTIVITÉ TOTALE DES FACTEURS (PTF)}

La productivité totale des facteurs peut être définie comme le rapport entre la production d'une firme et une mesure agrégée des inputs qu'elle emploie. Considérons une fonction de production Cobb-Douglas à deux facteurs de production, le travail $(L)$ et le capital $(K)$. La PTF d'une firme $i$ à la date $t$ s'écrit alors :

$$
P T F_{i t}=\frac{Y_{i t}}{L_{i t}^{\alpha} K_{i t}^{\beta}}
$$

En passant en logarithme, la PTF s'écrit :

In $P T F_{i t}=y_{i t}-\left(\beta l_{i t}+\beta_{k} k_{i t}\right)$ où $\mathrm{y}$, l et $\mathrm{k}$ sont le logarithme de la production, du travail et du capital.

Selon cette approche, la PTF est définie comme le résidu de Solow, c'est-à-dire la partie de la production qui n'est pas expliquée par l'utilisation des facteurs de production. II existe plusieurs méthodes pour estimer la PTF (voir Dovis, 2008, pages 13-67, pour une présentation exhaustive des méthodes de calcul de la PTF) : I'approche semi-paramétrique initiée Olley et Pakes (1996) compte parmi les plus utilisées.

L'approche d'Olley et Pakes (1996) estime le résidu de Solow en suivant une approche non paramétrique. L'estimation des coefficients de la fonction de production donne une évaluation de la PTF :

$\operatorname{In} P T F_{i t}=y_{i t}-\hat{\beta}_{l} I_{i t}-\hat{\beta}_{k} k_{i t}$

Dans la fonction de production ci-dessous, le terme d'erreur $\varepsilon_{i t}$ est décomposé en un choc de productivité $\omega_{i t}$ et un terme d'erreur $\eta_{i t}$ :

$y_{i t}=\beta_{0}+\beta l_{i t}+\beta_{k} k_{i t}+\omega_{i t}+\eta_{i t}$.

La méthode initiée par Olley et Pakes (OP) s'efforce de corriger les problèmes isolés dans le terme $\omega_{i t}$ le biais de simultanéité et le biais de sélection.

Le biais de simultanéité

Le biais de simultanéité provient du fait que l'entreprise, contrairement à l'économètre, observe le choc de productivité $\omega_{i t}$. Ainsi, le niveau des inputs est choisi simul- tanément à l'observation de certains chocs de productivité : le niveau des inputs et ces chocs de productivité non observables sont donc corrélés positivement. Pour corriger ce biais, OP suppose une relation croissante entre l'investissement et le niveau de productivité non observable pour un niveau donné du stock de capital. En inversant cette fonction, il est possible d'exprimer le niveau de productivité non observable comme une fonction de l'investissement et du stock de capital. Cette méthode suppose qu'il y a peu d'observations pour lesquelles l'investissement est nul. C'est le cas pour seulement $4 \%$ des données que nous utilisons. Levinsohn et Petrin (2003) suggèrent l'utilisation des dépenses d'électivité et des consommations intermédiaires pour tenir compte des chocs de productivité non observables.

$y_{i t}=\beta J_{i t}+\varphi\left(i_{i t}, k_{i t}\right)+\eta_{i t}$

$\operatorname{avec} \varphi\left(i_{i t}, k_{i t}\right)=\beta_{0}+\beta_{k} k+h\left(i_{i t}, k_{i t}\right)$

Une fois la fonction $\phi($.$) approchée par un développe-$ ment limité d'ordre 4 , le coefficient du facteur travail est alors estimé par la méthode des MCO.

\section{Le biais de sélection}

Le biais de sélection provient du fait que l'estimation des paramètres de la fonction de production s'appuie uniquement sur les entreprises qui restent sur le marché. Dans cette approche, les entreprises décident de se maintenir ou non sur un marché en fonction de leur niveau de productivité, dont une partie n'est pas observable. Si le niveau de productivité est supérieur à un certain seuil, l'entreprise reste sur le marché et choisit le niveau de ses inputs. Sinon, elle sort du marché et récupère la valeur de liquidation. Comme le profit est une fonction croissante du niveau de capital et du niveau de productivité, les entreprises avec un niveau de capital élevé résisteront mieux à un faible niveau de productivité. Le biais de sélection peut donc s'accompagner d'une corrélation négative entre capital et productivité au sein de l'échantillon. Pour corriger ce biais, OP estiment la probabilité de survie de l'entreprise à la date t qui dépend du capital et de l'investissement en $\mathrm{t}-1$. Cette probabilité de survie est estimée avec une régression probit. Le coefficient du capital est alors estimé en utilisant le coefficient du facteur travail, la fonction $\phi$ et la probabilité de survie. 


\section{La France vUe \\ du recessessjesj" de la populatios}

\section{www.insee fr}

Les résultats du recensement de la population mis à jour chaque année
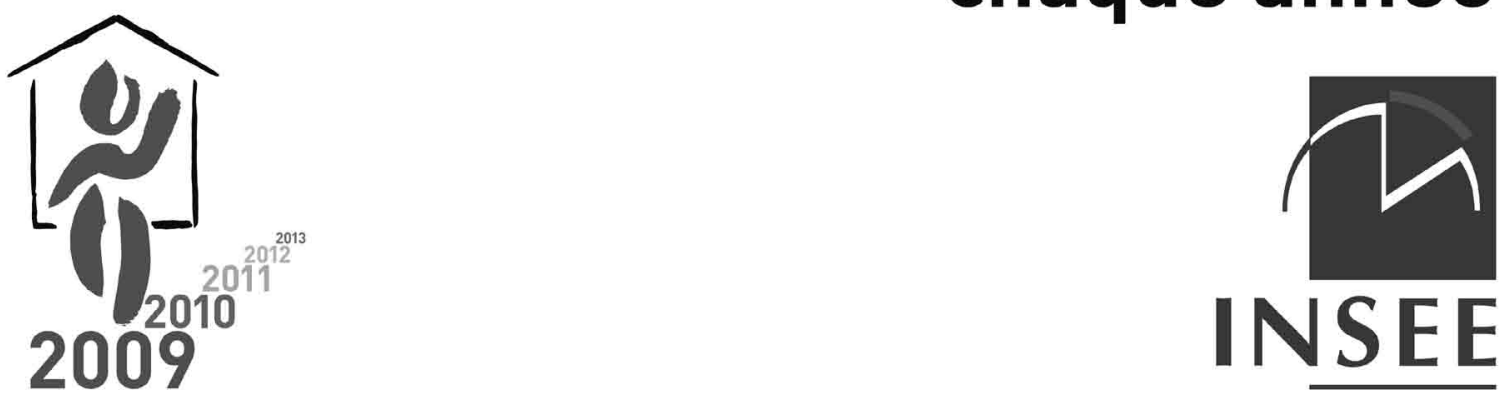